Article

\title{
Studies on Cytotoxic Constituents from the Leaves of Elaeagnus oldhamii Maxim. in Non-Small Cell Lung Cancer A549 Cells
}

Chi-Ren Liao ${ }^{1, \dagger}$, Yueh-Hsiung Kuo ${ }^{1,2}$, Yu-Ling Ho ${ }^{3, \dagger}$, Ching-Ying Wang ${ }^{1,4}$, Chang -Syun Yang ${ }^{1}$, Cheng-Wen Lin ${ }^{2,4}$ and Yuan-Shiun Chang ${ }^{1, *}$

1 Department of Chinese Pharmaceutical Sciences and Chinese Medicine Resources, College of Pharmacy, China Medical University, Taichung 40402, Taiwan

2 Department of Biotechnology, College of Health Science, Asia University, Wufeng, Taichung, 41354, Taiwan

3 Department of Nursing, Hung Kuang University, Taichung 43302, Taiwan

4 Department of Medical Laboratory Science and Biotechnology, China Medical University, Taichung 40402, Taiwan

$\dagger$ These two authors contributed equally to this work.

* Authors to whom correspondence should be addressed;

E-Mails: cwlin@mail.cmu.edu.tw (C.-W.L.); yschang@mail.cmu.edu.tw (Y.-S.C.);

Tel.: +886-4-2205-3366 (ext. 7210) (C.-W.L.); +886-4-2205-3366 (ext. 5502) (Y.-S.C.);

Fax: +886-4-2205-7414 (C.-W.L.); +886-4-2208-3362 (Y.-S.C.).

Received: 29 April 2014; in revised form: 15 June 2014 / Accepted: 25 June 2014 /

Published: 4 July 2014

Abstract: Elaeagnus oldhamii Maxim. is a commonly used traditional herbal medicine. In Taiwan the leaves of E. oldhamii Maxim. are mainly used for treating lung disorders. Twenty five compounds were isolated from the leaves of E. oldhamii Maxim. in the present study. These included oleanolic acid (1), 3-O-(Z)-coumaroyl oleanolic acid (2), 3-O-(E)-coumaroyl oleanolic acid (3), 3-O-caffeoyl oleanolic acid (4), ursolic acid (5), 3-O-(Z)-coumaroyl ursolic acid (6), 3-O-(E)-coumaroyl ursolic acid (7), 3-O-caffeoyl ursolic

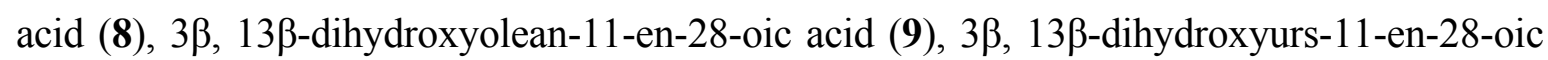
acid (10), uvaol (11), betulin (12), lupeol (13), kaempferol (14), aromadendrin (15), epigallocatechin (16), cis-tiliroside (17), trans-tiliroside (18), isoamericanol B (19), trans-p-coumaric acid (20), protocatechuic acid (21), salicylic acid (22), trans-ferulic acid (23), syringic acid (24) and 3-O-methylgallic acid (25). Of the 25 isolated compounds, 21 
compounds were identified for the first time in E. oldhamii Maxim. These included compounds 1, 4, 5 and 8-25. These 25 compounds were evaluated for their inhibitory activity against the growth of non-small cell lung cancer A549 cells by the MTT assay, and the corresponding structure-activity relationships were discussed. Among these 25 compounds, compound 6 displayed the best activity against the A549 cell line in vitro $\left(\mathrm{CC}_{50}=8.56 \pm 0.57 \mu \mathrm{g} / \mathrm{mL}\right.$, at $48 \mathrm{~h}$ of MTT asssay). Furthermore, compound 2, 4, 8 and 18 exhibited in vitro cytotoxicity against the $\mathrm{A} 549$ cell line with the $\mathrm{CC}_{50}$ values of less than $20 \mu \mathrm{g} / \mathrm{mL}$ at $48 \mathrm{~h}$ of MTT asssay. These five compounds $2,4,6,8$ and 18 exhibited better cytotoxic activity compared with cisplatin (positive control, $\mathrm{CC}_{50}$ value of $14.87 \pm 1.94 \mu \mathrm{g} / \mathrm{mL}$, at $48 \mathrm{~h}$ of MTT asssay). The result suggested that the five compounds might be responsible for its clinical anti-lung cancer effect.

Keywords: traditional herbal medicine; Elaeagnus oldhamii Maxim.; non-small cell lung cancer A549 cells; cytotoxicity; MTT assay

\section{Introduction}

There are about 90 species of Elaeagnus around the world and nine species can be found in Taiwan [1]. Many species of Elaeagnus are used as folk medicinal plants, e.g., E. umbellate [2], E. pungens [3], E. angustifolia [4,5] and E. multiflora [6]. The cytotoxic activities of several species of Elaeagnus have been evaluated in previous studies, e.g., E. angustifolia [7], E. umbellate [8], E. pungens [9] and E. glabra [10]. Triterpenoid [11-13], flavonoid [14-16], lignanoid [17-19] and benzenoid [20-22] compounds are four of the major classes of bioactive compounds used for their anti-tumor properties. Furthermore, triterpenoid, flavonoid, lignanoid and benzenoid compounds were isolated from several species of Elaeagnus, e.g., E. pungens [23], E. bockii [24], E. lanceolata, [25,26] and E. angustifolia [27] in previous studies.

Lung cancer is one of the cancers which commonly causes deaths all over the world. In addition, lung cancer is the first most lethal cancer type and the third rate of occurrence cancer in Taiwan, respectively [28,29]. Traditionally, the leaves of E. oldhamii Maxim. were mainly used for treating lung disorders in Taiwan, such as coughs, asthma and pulmonary abscesses. Based on our interest in the anti-lung cancer activity of this plant, EOM (methanol extract of E. oldhamii Maxim.) and three partitioned fractions of the methanol extract of E. oldhamii Maxim., including EOE (EtOAc-soluble fraction), EOB (BuOH-soluble fraction) and EOW (water-soluble fraction) had been evaluated for their inhibitory activity against the growth of non-small cell lung cancer A549 cells by MTT assays in the present study. Non-small cell lung cancer (NSCLC) accounts for $85 \%$ of all cases of lung cancer, and the A549 cells line is a kind of non-small cell lung cancer cell which was often applied to evaluate cytotoxic activity in vitro [30,31]. The results showed that EtOAc-soluble fraction exhibited the best activity against the $\mathrm{A} 549$ cell line in vitro $\left(\mathrm{CC}_{50}=45.15 \pm 1.10\right.$, at $48 \mathrm{~h}$ of MTT asssay). Based on our interest in the chemical components of this plant, the EtOAc-soluble fraction was further fractioned and purified. A total of 25 compounds (Figure 1) were isolated from the leaves of E. oldhamii Maxim. Twenty one of these 25 compounds, including compound 1, 4, 5 and 8-25, were found for the first 
time in the leaves of E. oldhamii Maxim. Furthermore, eleven of these compounds, including 2-4, 6-10, 13, 18 and 19 were evaluated for the first time regarding their cytotoxic activity in non-small cell lung cancer A549 cells by MTT assay. The isolation procedure, the ${ }^{1} \mathrm{H}$ - and ${ }^{13} \mathrm{C}-\mathrm{NMR}$ spectra and the cytotoxic activity of these total 25 isolates are described herein.

Figure 1. The chemical structures of compounds 1-25.

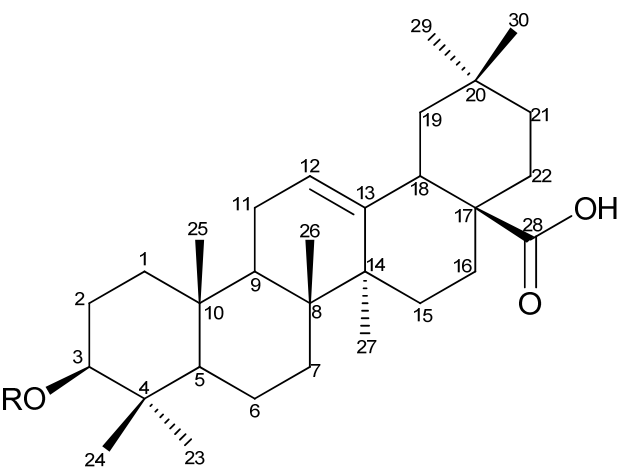

$1 \mathrm{R}=\quad \mathrm{H}$

$2 \mathrm{R}=$<smiles>CC(=O)/C=C/c1ccc(O)cc1</smiles>

$3 \mathrm{R}=$<smiles>CC(=O)C=Cc1ccc(O)cc1</smiles>

$4 \mathrm{R}=$<smiles>CC(=O)C=Cc1ccc(O)cc1</smiles>

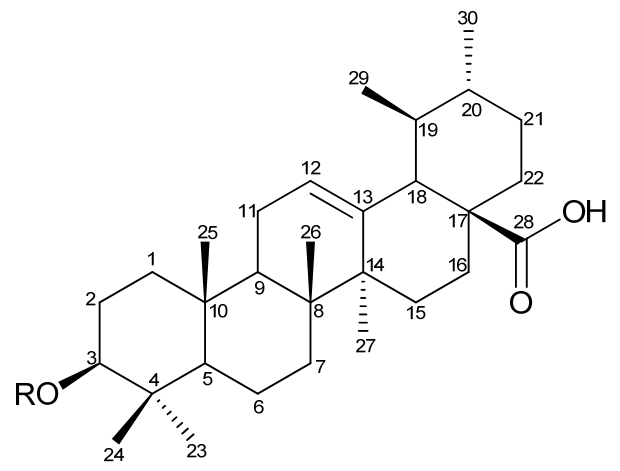

$5 \mathrm{R}=\quad \mathrm{H}$

$6 \mathrm{R}=$<smiles>CC(=O)C=Cc1ccc(O)cc1</smiles>

$7 \mathrm{R}=$<smiles>CC(=O)C=Cc1ccc(O)cc1</smiles>

$8 \mathrm{R}=$<smiles>CC(=O)/C=C/c1ccc(O)cc1</smiles>

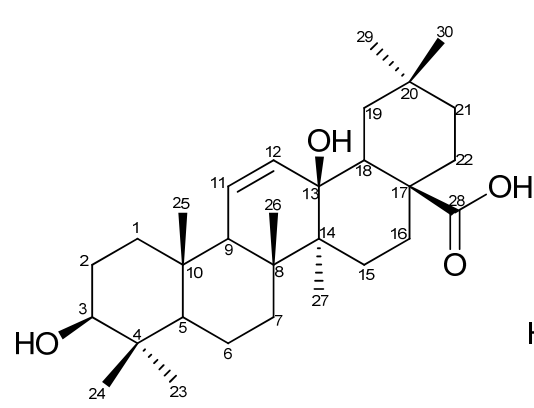

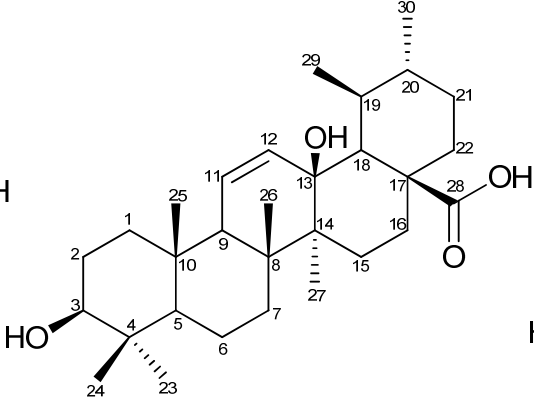

10

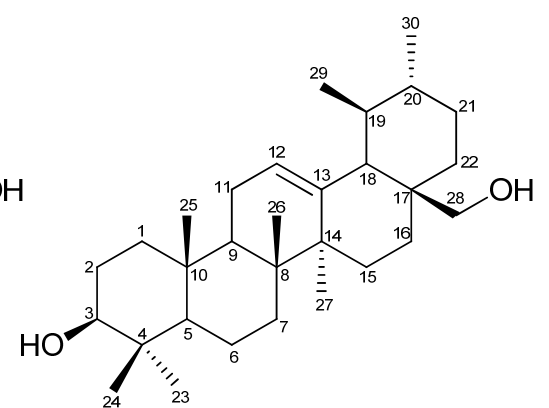

11 
Figure 1. Cont.

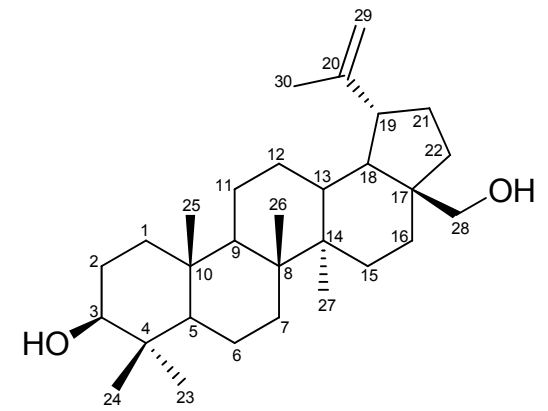

12

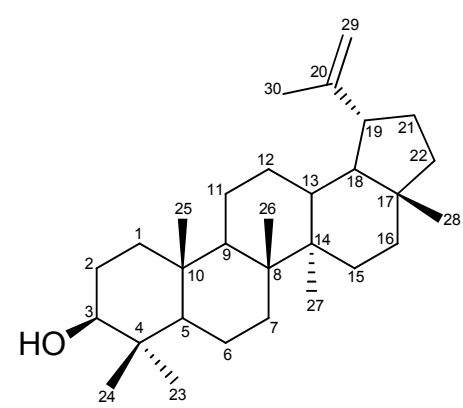

13<smiles>O=c1c(O)c(-c2ccc(O)cc2)oc2cc(O)cc(O)c12</smiles>

14

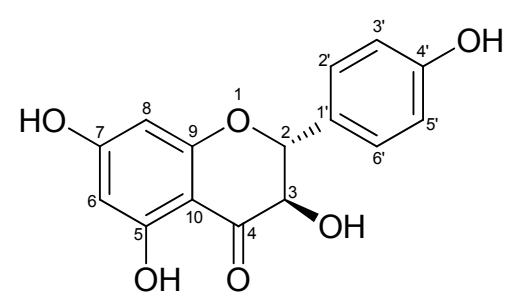

15<smiles></smiles>

16<smiles>O=C(C=[Te]c1ccc(O)cc1)OC[C@H]1OC(Oc2c(-c3ccc(O)cc3)oc3cc(O)cc(O)c3c2=O)[C@H](O)[C@@H](O)[C@H]1O</smiles>

17<smiles>O=C(/C=C/c1ccc(O)cc1)OC[C]1O[C@H](Oc2c(-c3ccc(O)cc3)oc3cc(O)cc(O)c3c2=O)[C@H](O)C(O)[C@@H]1O</smiles>

18<smiles>COc1cc(C2Oc3cc(/C=C/CO)ccc3O[C@@H]2C)ccc1O</smiles>

19<smiles>O=C(O)/C=C/c1ccc(O)cc1</smiles>

20<smiles>O=C(O)c1ccc(O)c(O)c1</smiles>

21<smiles>O=C(O)c1ccccc1O</smiles>

22<smiles>COc1cc(/C=C/C(=O)O)ccc1O</smiles>

23<smiles>COc1cc(C(=O)O)cc(OC)c1O</smiles>

24<smiles>COc1cc(C(=O)O)cc(O)c1O</smiles>

25 


\section{Results and Discussion}

The ${ }^{1} \mathrm{H}$ - and ${ }^{13} \mathrm{C}-\mathrm{NMR}$ spectra of the 25 compounds isolated in present study (Tables $1-3$ and Section 3.4) including oleanolic acid (1) [32], 3-O-(Z)-coumaroyl oleanolic acid (2) [33], 3-O-(E)coumaroyl oleanolic acid (3) [34], 3-O-caffeoyl oleanolic acid (4) [35], ursolic acid (5) [36], 3-O-(Z)coumaroyl ursolic acid (6) [35], 3-O-(E)-coumaroyl ursolic acid (7) [36], 3-O-caffeoyl ursolic acid (8) [37],

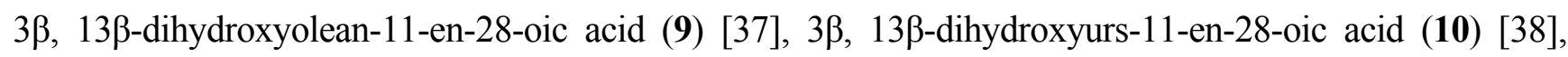
uvaol (11) [39], betulin (12) [40], lupeol (13) [41], kaempferol (14) [42], aromadendrin (15) [43], epigallocatechin (16) [44], cis-tiliroside (17) [45], trans-tiliroside (18) [46], isoamericanol B (19) [47], trans-p-coumaric acid (20) [47], protocatechuic acid (21) [48], salicylic acid (22) [49], trans-ferulic acid (23) [50], syringic acid (24) [51] and 3-O-methylgallic acid (25) [52] were compared with the spectral data reported in the literature and their structures were thus confirmed.

Non-small cell lung cancer (NSCLC) accounts for $85 \%$ of all lung cancer cases, and A549 cell line is one kind of non-small cell lung cancer cells which was used to evaluate cytotoxic activity in vitro [30,31]. Therefore, we chose the A549 cell line to evaluate cytotoxic activity of these 25 compounds. In this study, four triterpenoid compounds (compounds 2, 4, 6 and 8) and one flavanoid compound (compound 18) isolated from the crude extract displayed excellent cytotoxic activity against the A549 cell line with $\mathrm{CC}_{50}$ values of less than $20 \mu \mathrm{g} / \mathrm{mL}$.

Oleanolic acid and ursolic acid exhibited good cytotoxic activity against the A549 cell line in previous studies [53-55]. However, compound 2, 4, 6 and 8 were oleanolic acid and ursolic acid 3-O- derivatives, and these four compounds showed better cytotoxic activity than oleanolic acid (compound 1) and ursolic acid (compound 5) in the present study. The $\mathrm{CC}_{50}$ values of compound $\mathbf{1}$ and compound $\mathbf{5}$ were $52.97 \pm 1.22$ and $154.73 \pm 7.86$ at $48 \mathrm{~h}$ of MTT assay, and compound 2, 4, 6 and $\mathbf{8}$ were $9.46 \pm 1.50,13.76 \pm 3.45,8.56 \pm 0.57$ and $15.70 \pm 1.24$ at $48 \mathrm{~h}$ of MTT assay respectively (Table 4). Furthermore, regard to function groups of these four compounds, compound $\mathbf{2}$ and $\mathbf{6}$ were oleanolic acid and ursolic acid 3-O-linkage to cis-coumaryl, and compound $\mathbf{4}$ and $\mathbf{8}$ were 3-O-linkage to caffeoyl. In addition, oleanolic acid and ursolic acid 3-O-linkage to cis-coumaroyl (compounds 2 and 6) exhibited better cytotoxic activity than 3-O-linkage to trans-coumaroyl (compounds 3 and 7). The $\mathrm{CC}_{50}$ value of compound $\mathbf{2}$ and $\mathbf{3}$ were $9.46 \pm 1.50$ and $65.21 \pm 7.15$, and compounds $\mathbf{6}$ and 7 were 8.56 \pm 0.57 and $79.59 \pm 5.77$ repectively (Table 4). Therefore, we believe that 3 - $O$-linkage to cis-coumaroyl and caffeoyl of oleanolic acid and ursolic acid were two active functional groups responsible for the cytotoxic activity against the growth of non-small cell lung cancer A549 cells according to the MTT assay.

Kaempferol was investigated for its cytotoxic activity against the A549 cell line in a previous study [56]. Compound 17 was a kaempferol derivative and exhibited better cytotoxic activity than kaempferol (compound 14) in present study. The $\mathrm{CC}_{50}$ value of compound $\mathbf{1 4}$ was $113.48 \pm 5.32$, and that of compound 17 was $18.82 \pm 3.64$ at $48 \mathrm{~h}$ of MTT assay, respectively (Table 4). Furthermore, with regard to the functional groups of compound 17, it has a kaempferol 3-O-linkage to D-glucose and a 6"-O-linkage to a cis-coumaroyl moiety. In addition, the kaempferol 6"-O-linkage to a cis-coumaroyl group (compound 17) exhibited better cytotoxic activity than a $6 "-O$-linkage to a trans-coumaroyl group (compound 18). The $\mathrm{CC}_{50}$ value of compound 17 was $18.82 \pm 3.64$, and that of compound 18 was $144.74 \pm 5.37$ at $48 \mathrm{~h}$ MTT assay, respectively (Table 4). Therefore, we also believe that in this 
case the 3-O-linkage to D-glucose and 6"-O-linkage to the cis-coumaroyl were the active functional groups that provide better cytotoxic activity against the growth of non-small cell lung cancer A549 cells according to the MTT assay.

Table 1. ${ }^{13} \mathrm{C}-\mathrm{NMR}$ spectroscopic data (500 MHz) of compounds 1-13.

\begin{tabular}{|c|c|c|c|c|c|c|}
\hline Position & $1^{d}$ & $2^{b}$ & $3^{c}$ & $4^{d}$ & $5^{d}$ & $6^{b}$ \\
\hline 1 & 39.4 & 37.2 & 39.3 & 38.6 & 39.4 & 38.0 \\
\hline 2 & 28.5 & 23.1 & 24.1 & 24.1 & 28.4 & 28.2 \\
\hline 3 & 78.5 & 81.4 & 85.7 & 80.9 & 78.5 & 81.4 \\
\hline 4 & 39.8 & 38.0 & 39.4 & 38.7 & 39.8 & 38.5 \\
\hline 5 & 56.2 & 55.5 & 56.6 & 56.1 & 56.2 & 55.5 \\
\hline 6 & 19.2 & 18.4 & 19.6 & 19.0 & 19.1 & 18.4 \\
\hline 7 & 33.7 & 32.7 & 33.7 & 33.5 & 33.9 & 33.0 \\
\hline 8 & 40.2 & 39.5 & 40.8 & 40.2 & 40.3 & 39.7 \\
\hline 9 & 48.6 & 47.7 & 49.1 & 48.4 & 48.4 & 48.2 \\
\hline 10 & 37.8 & 38.3 & 39.4 & 37.7 & 37.8 & 37.1 \\
\hline 11 & 24.2 & 23.7 & 24.8 & 24.6 & 34.0 & 23.5 \\
\hline 12 & 123.0 & 122.8 & 123.5 & 122.8 & 126.0 & 126.0 \\
\hline 13 & 145.2 & 143.8 & 146.4 & 144.4 & 139.6 & 138.2 \\
\hline 14 & 42.6 & 41.8 & 43.1 & 42.6 & 42.9 & 42.1 \\
\hline 15 & 28.7 & 27.9 & 28.9 & 28.8 & 29.0 & 28.3 \\
\hline 16 & 24.2 & 22.9 & 24.1 & 24.2 & 25.3 & 24.2 \\
\hline 17 & 47.1 & 46.8 & 47.8 & 47.2 & 48.4 & 47.6 \\
\hline 18 & 42.4 & 41.1 & 42.9 & 42.5 & 53.9 & 52.7 \\
\hline 19 & 46.9 & 46.1 & 47.4 & 47.0 & 39.7 & 39.2 \\
\hline 20 & 31.4 & 30.9 & 29.4 & 31.5 & 39.7 & 39.0 \\
\hline 21 & 34.7 & 34.0 & 35.0 & 34.8 & 31.4 & 30.8 \\
\hline 22 & 33.6 & 32.7 & 31.8 & 32.6 & 37.6 & 37.0 \\
\hline 23 & 29.2 & 28.3 & 28.9 & 28.7 & 29.2 & 29.9 \\
\hline 24 & 17.0 & 15.6 & 17.9 & 17.9 & 16.9 & 17.0 \\
\hline 25 & 16.0 & 16.9 & 17.2 & 15.9 & 16.0 & 15.8 \\
\hline 26 & 17.9 & 17.4 & 18.4 & 17.6 & 17.8 & 17.2 \\
\hline 27 & 26.6 & 26.1 & 26.6 & 26.7 & 24.2 & 23.8 \\
\hline 28 & 180.6 & 184.3 & 182.0 & 180.9 & 180.2 & 184.1 \\
\hline 29 & 33.7 & 33.3 & 33.9 & 33.8 & 17.8 & 17.3 \\
\hline 30 & 24.1 & 23.6 & 24.2 & 23.8 & 21.8 & 21.4 \\
\hline $1^{\prime}$ & - & 167.0 & 169.8 & 167.8 & - & 167.0 \\
\hline $2^{\prime}$ & - & 117.7 & 116.0 & 116.4 & - & 117.7 \\
\hline $3^{\prime}$ & - & 143.7 & 145.5 & 145.4 & - & 143.8 \\
\hline $4^{\prime}$ & - & 127.5 & 127.5 & 127.4 & - & 127.5 \\
\hline $5^{\prime}$ & - & 132.4 & 131.2 & 116.0 & - & 132.4 \\
\hline $6^{\prime}$ & - & 115.3 & 117.0 & 146.2 & - & 115.3 \\
\hline $7^{\prime}$ & - & 157.1 & 161.3 & 149.9 & - & 157.2 \\
\hline $8^{\prime}$ & - & 115.3 & 117.0 & 117.4 & - & 115.3 \\
\hline $9^{\prime}$ & - & 132.4 & 131.2 & 122.5 & - & 132.4 \\
\hline
\end{tabular}


Table 1. Cont.

\begin{tabular}{|c|c|c|c|c|c|c|c|}
\hline Position & $7^{c}$ & $8^{c}$ & $9^{b}$ & $10^{b}$ & $11^{b}$ & $12^{b}$ & $13^{b}$ \\
\hline 1 & 39.3 & 37.9 & 37.5 & 38.4 & 39.0 & 38.9 & 38.9 \\
\hline 2 & 29.3 & 24.5 & 23.8 & 23.0 & 27.5 & 27.6 & 29.9 \\
\hline 3 & 85.7 & 81.0 & 79.0 & 79.1 & 79.2 & 79.2 & 79.2 \\
\hline 4 & 40.6 & 37.6 & 38.5 & 39.1 & 38.2 & 38.9 & 39.1 \\
\hline 5 & 56.6 & 56.2 & 53.4 & 55.0 & 55.4 & 55.5 & 55.5 \\
\hline 6 & 19.6 & 19.8 & 18.2 & 17.9 & 18.5 & 18.5 & 18.6 \\
\hline 7 & 34.3 & 33.5 & 29.9 & 29.9 & 33.0 & 34.5 & 34.5 \\
\hline 8 & 41.0 & 40.3 & 41.6 & 41.9 & 40.2 & 39.8 & 41.1 \\
\hline 9 & 50.0 & 47.6 & 50.7 & 53.3 & 47.9 & 50.6 & 50.7 \\
\hline 10 & 38.2 & 36.6 & 34.6 & 36.6 & 37.1 & 37.5 & 37.4 \\
\hline 11 & 24.3 & 24.2 & 136.1 & 133.7 & 23.6 & 21.0 & 21.2 \\
\hline 12 & 126.8 & 123.0 & 127.1 & 129.1 & 125.3 & 25.4 & 23.6 \\
\hline 13 & 139.9 & 145.1 & 90.1 & 89.9 & 138.9 & 37.4 & 38.3 \\
\hline 14 & 43.5 & 42.7 & 41.8 & 42.2 & 42.3 & 41.1 & 43.1 \\
\hline 15 & 29.4 & 28.6 & 27.4 & 27.2 & 26.2 & 27.3 & 27.7 \\
\hline 16 & 25.4 & 26.4 & 25.6 & 25.8 & 23.6 & 29.4 & 35.8 \\
\hline 17 & 49.3 & 46.9 & 44.2 & 45.3 & 37.1 & 48.0 & 42.2 \\
\hline 18 & 54.4 & 48.5 & 55.0 & 60.8 & 54.2 & 42.9 & 48.5 \\
\hline 19 & 40.8 & 39.0 & 36.6 & 38.5 & 39.6 & 49.0 & 48.2 \\
\hline 20 & 40.6 & 38.9 & 39.2 & 40.5 & 39.6 & 150.7 & 151.2 \\
\hline 21 & 31.9 & 31.4 & 31.4 & 31.5 & 30.8 & 30.0 & 30.1 \\
\hline 22 & 38.2 & 36.9 & 31.7 & 31.6 & 35.4 & 34.2 & 40.2 \\
\hline 23 & 31.0 & 28.5 & 28.0 & 28.0 & 28.3 & 28.2 & 28.2 \\
\hline 24 & 17.8 & 17.1 & 15.1 & 15.1 & 15.9 & 15.6 & 14.8 \\
\hline 25 & 17.4 & 15.9 & 17.9 & 16.3 & 15.8 & 16.3 & 16.3 \\
\hline 26 & 17.9 & 17.7 & 19.2 & 19.1 & 17.0 & 16.2 & 16.2 \\
\hline 27 & 24.6 & 24.0 & 18.5 & 18.1 & 23.5 & 14.7 & 15.6 \\
\hline 28 & 181.8 & 179.1 & 180.2 & 180.1 & 70.1 & 60.8 & 18.2 \\
\hline 29 & 18.5 & 17.7 & 33.5 & 18.0 & 17.6 & 109.9 & 109.5 \\
\hline 30 & 21.7 & 23.9 & 27.2 & 19.4 & 21.5 & 19.3 & 19.5 \\
\hline $1^{\prime}$ & 169.8 & 167.3 & - & - & - & - & - \\
\hline $2^{\prime}$ & 116.0 & 116.2 & - & - & - & - & - \\
\hline $3^{\prime}$ & 146.4 & 145.5 & - & - & - & - & - \\
\hline $4^{\prime}$ & 127.5 & 127.2 & - & - & - & - & - \\
\hline $5^{\prime}$ & 131.2 & 115.2 & - & - & - & - & - \\
\hline $6^{\prime}$ & 117.0 & 146.5 & - & - & - & - & - \\
\hline $7^{\prime}$ & 161.3 & 148.9 & - & - & - & - & - \\
\hline $8^{\prime}$ & 117.0 & 116.4 & - & - & - & - & - \\
\hline $9^{\prime}$ & 131.2 & 122.5 & - & - & - & - & - \\
\hline
\end{tabular}

${ }^{b}$ In chloroform- $d ;{ }^{c}$ In methanol- $d_{4} ;{ }^{d}$ In pyridine- $d_{5}$. 
Table 2. ${ }^{13} \mathrm{C}-\mathrm{NMR}$ spectroscopic data $(500 \mathrm{MHz})$ of compounds 14-18.

\begin{tabular}{|c|c|c|c|c|c|}
\hline Position & $14^{c}$ & $15^{c}$ & $16^{c}$ & $17^{a}$ & $18^{b}$ \\
\hline 1 & - & - & - & - & - \\
\hline 2 & 148.2 & 85.1 & 79.5 & 158.8 & 159.5 \\
\hline 3 & 137.3 & 73.8 & 67.1 & 135.3 & 135.4 \\
\hline 4 & 177.5 & 198.5 & 30.7 & 179.1 & 179.4 \\
\hline 5 & 162.7 & 165.5 & 157.6 & 158.0 & 158.5 \\
\hline 6 & 99.4 & 97.6 & 96.2 & 99.8 & 100.1 \\
\hline 7 & 165.8 & 169.4 & 157.6 & 165.4 & 166.0 \\
\hline 8 & 94.6 & 96.6 & 95.8 & 94.8 & 95.0 \\
\hline 9 & 158.4 & 164.7 & 157.2 & 162.9 & 163.1 \\
\hline 10 & 104.7 & 101.9 & 99.9 & 105.5 & 105.7 \\
\hline $1^{\prime}$ & 123.9 & 129.5 & 131.6 & 122.4 & 122.9 \\
\hline $2^{\prime}$ & 130.8 & 130.5 & 107.0 & 132.4 & 132.4 \\
\hline $3^{\prime}$ & 116.5 & 116.3 & 146.2 & 116.1 & 116.2 \\
\hline $4^{\prime}$ & 140.7 & 159.4 & 133.0 & 161.0 & 161.5 \\
\hline $5^{\prime}$ & 116.5 & 116.3 & 146.2 & 116.1 & 116.2 \\
\hline $6^{\prime}$ & 130.8 & 130.5 & 107.0 & 132.4 & 132.4 \\
\hline $1^{\prime \prime}$ & - & - & - & 104.5 & 104.2 \\
\hline $2^{\prime \prime}$ & - & - & - & 75.5 & 75.9 \\
\hline $3 "$ & - & - & - & 71.1 & 71.9 \\
\hline 4" & - & - & - & 75.3 & 75.9 \\
\hline $5^{\prime \prime}$ & - & - & - & 78.1 & 78.1 \\
\hline $6^{\prime \prime}$ & - & - & - & 63.9 & 64.5 \\
\hline $1 " '$ & - & - & - & 127.3 & 127.2 \\
\hline $2^{\prime \prime \prime}$ & - & - & - & 133.8 & 133.9 \\
\hline $3 " '$ & - & - & - & 115.7 & 115.9 \\
\hline $4 " '$ & - & - & - & 159.7 & 161.3 \\
\hline $5^{\prime \prime \prime}$ & - & - & - & 115.7 & 115.9 \\
\hline 6"' & - & - & - & 133.8 & 133.9 \\
\hline 7"' & - & - & - & 144.5 & 146.7 \\
\hline 8"' & - & - & - & 116.3 & 116.9 \\
\hline 9"' & - & - & - & 166.3 & 169.0 \\
\hline
\end{tabular}

${ }^{a}$ In acetone- $d_{6} ;{ }^{b}$ In chloroform- $d ;{ }^{c}$ In methanol- $d_{4}$.

Table 3. ${ }^{13} \mathrm{C}-\mathrm{NMR}$ spectroscopic data (500 MHz) of compounds 19-25.

\begin{tabular}{clllllll}
\hline Position & $\mathbf{1 9}^{\boldsymbol{a}}$ & $\mathbf{2 0}^{\boldsymbol{c}}$ & $\mathbf{2 1}^{\boldsymbol{c}}$ & $\mathbf{2 2}^{\boldsymbol{a}}$ & $\mathbf{2 3}^{\boldsymbol{c}}$ & $\mathbf{2 4}^{\boldsymbol{c}}$ & $\mathbf{2 5}^{\boldsymbol{c}}$ \\
\hline 1 & 129.4 & 127.4 & 123.2 & 114.3 & 128.1 & 120.6 & 122.4 \\
2 & 111.3 & 131.2 & 117.5 & 163.3 & 111.8 & 106.8 & 106.5 \\
3 & 148.5 & 116.9 & 145.7 & 118.3 & 150.6 & 147.4 & 146.3 \\
4 & 147.6 & 161.3 & 150.8 & 136.6 & 149.5 & 140.1 & 140.5 \\
5 & 117.9 & 116.9 & 115.8 & 120.2 & 116.6 & 147.4 & 149.2 \\
6 & 120.3 & 131.2 & 123.7 & 131.7 & 124.0 & 106.8 & 112.5 \\
7 & 78.1 & 146.7 & 167.8 & 173.8 & 146.7 & 167.2 & 170.5 \\
8 & 73.8 & 116.0 & - & - & 116.6 & - & - \\
9 & 13.7 & 171.3 & - & - & 171.5 & - & - \\
\hline
\end{tabular}


Table 3. Cont.

\begin{tabular}{clcccccc}
\hline Position & $\mathbf{1 9}^{a}$ & $\mathbf{2 0}^{\boldsymbol{c}}$ & $\mathbf{2 1}^{\boldsymbol{c}}$ & $\mathbf{2 2}^{\boldsymbol{c}}$ & $\mathbf{2 3}^{c}$ & $\mathbf{2 4}^{c}$ & $\mathbf{2 5}^{c}$ \\
\hline $1^{\prime}$ & 56.5 & - & - & - & - & - & - \\
$2^{\prime}$ & 132.8 & - & - & - & - & - & - \\
$3^{\prime}$ & 118.3 & - & - & - & - & - & - \\
$4^{\prime}$ & 143.9 & - & - & - & - & - & - \\
$5^{\prime}$ & 142.6 & - & - & - & - & - & - \\
$6^{\prime}$ & 115.9 & - & - & - & - & - & - \\
$7^{\prime}$ & 123.5 & - & - & - & - & - & - \\
$8^{\prime}$ & 129.6 & - & - & - & - & - & - \\
$9^{\prime}$ & 131.5 & - & - & - & - & - & - \\
$\mathrm{OCH}_{3}$ & 59.8 & & & & 56.6 & 55.9 & 56.8 \\
\hline
\end{tabular}

${ }^{a}$ In acetone- $d_{6} ;{ }^{c}$ In methanol- $d_{4}$.

Table 4. Cytotoxicities of compound 1-25 from E. oldhamii Maxim on A549 cell line.

\begin{tabular}{|c|c|c|c|}
\hline \multirow{2}{*}{ Sample Code } & \multirow{2}{*}{ Compound Name } & \multicolumn{2}{|c|}{$\mathrm{CC}_{50}(\mu \mathrm{g} / \mathrm{mL})$} \\
\hline & & $24 \mathrm{~h}$ & $48 \mathrm{~h}$ \\
\hline Positive control & Cisplatin & $132.05 \pm 1.46$ & $14.87 \pm 1.94$ \\
\hline 1 & Oleanolic acid & $64.86 \pm 3.90$ & $52.97 \pm 1.22$ \\
\hline 2 & 3-O-(Z)-coumaroyl oleanolic acid & $9.23 \pm 1.73$ & $9.46 \pm 1.50$ \\
\hline 3 & 3-O-(E)-coumaroyl oleanolic acid & $135.12 \pm 3.08$ & $65.21 \pm 7.15$ \\
\hline 4 & 3-O-caffeoyl oleanolic acid & $12.35 \pm 1.52$ & $13.76 \pm 3.45$ \\
\hline 5 & Ursolic acid & $153.37 \pm 3.64$ & $154.73 \pm 7.86$ \\
\hline 6 & 3-O-(Z)-coumaroyl ursolic acid & $13.06 \pm 2.26$ & $8.56 \pm 0.57$ \\
\hline 7 & 3-O-(E)-coumaroyl ursolic acid & $121.53 \pm 9.68$ & $79.59 \pm 5.77$ \\
\hline 8 & 3-O-caffeoyl ursolic acid & $157.98 \pm 5.02$ & $15.70 \pm 1.24$ \\
\hline 9 & $3 \beta, 13 \beta$-Dihydroxyolean-11-en-28-oic acid & $92.71 \pm 3.89$ & $70.61 \pm 8.27$ \\
\hline 10 & $3 \beta, 13 \beta$-Dihydroxyurs-11-en-28-oic acid & $176.81 \pm 6.33$ & $156.20 \pm 8.10$ \\
\hline 11 & Uvaol & $169.45 \pm 9.47$ & $163.36 \pm 7.57$ \\
\hline 12 & Betulin & $>500$ & $>500$ \\
\hline 13 & Lupeol & $142.70 \pm 4.99$ & $119.30 \pm 5.28$ \\
\hline 14 & Kaempferol & $129.15 \pm 5.16$ & $113.48 \pm 5.32$ \\
\hline 15 & Aromadendrin & $76.66 \pm 4.35$ & $55.67 \pm 2.37$ \\
\hline 16 & Epigallocatechin & $193.77 \pm 3.98$ & $118.92 \pm 4.25$ \\
\hline 17 & cis-Tiliroside & $68.05 \pm 1.46$ & $18.82 \pm 3.64$ \\
\hline 18 & trans-Tiliroside & $149.90 \pm 4.14$ & $144.74 \pm 5.37$ \\
\hline 19 & Isoamericanol B & $>500$ & $126.97 \pm 4.83$ \\
\hline 20 & trans-p-Coumaric acid & $>500$ & $>500$ \\
\hline 21 & Protocatechuic acid & $>500$ & $>500$ \\
\hline 22 & Salicylic acid & $>500$ & $103.64 \pm 4.59$ \\
\hline 23 & trans-Ferulic acid & $78.09 \pm 5.78$ & $60.42 \pm 5.00$ \\
\hline 24 & Syringic acid & $73.41 \pm 2.92$ & $77.66 \pm 4.04$ \\
\hline 25 & 3-O-methylgallic acid & $>500$ & $180.60 \pm 8.58$ \\
\hline MOM & & $84.67 \pm 0.37$ & $89.98 \pm 7.03$ \\
\hline MOE & & $52.78 \pm 0.78$ & $45.15 \pm 1.10$ \\
\hline MOB & & $192.46 \pm 6.54$ & $166.38 \pm 12.65$ \\
\hline MOW & & $100.91 \pm 1.01$ & $136.47 \pm 3.96$ \\
\hline
\end{tabular}




\section{Experimental}

\subsection{General}

The 1D- and 2D-NMR spectra were recorded with a Bruker DRX-500 FT-NMR (Taichung, Taiwan) spectrometer. HPLC chromatograms were obtained with an LC-6A instrument and an IOTA-2 RI-detector (Shimadzu, Kyoto, Japan). Semi-preparative NP-HPLC column chromatography was performed using MonoChrom Si gel $(250 \mathrm{~mm} \times 10 \mathrm{~mm}$ i.d., $5 \mu \mathrm{m}$, MetaChem, Torrance, CA, USA), and semi-preparative RP-HPLC column chromatography was performed using a Discovery ${ }^{\circledR}$ C18 column $(250 \mathrm{~mm} \times 10 \mathrm{~mm}$ i.d., $5 \mu \mathrm{m}$, Supelco, Bellefonte, PA, USA), TLC was performed with aluminum pre-coated Si plates (Merck, Darmstadt, Germany), Silica gel (63-200 $\mu \mathrm{m})$ and Sephadex ${ }^{\circledR}$ LH-20 was $(25-100 \mu \mathrm{m})$ were purchased from Merck. A multiscan microplate reader (VersaMax ELISA Microplate Reader, Taipei, Taiwan) was used for the MTT assays. The solvents used in the study for open column isolation (Sephadex LH 20 and silica gel column), such as $n$-hexane, chloroform, ethyl acetate, acetone and methanol were ACS grade and purchased from Merck. HPLC grade $n$-hexane, chloroform, ethyl acetate, acetone, methanol, acetonitrile and ammonium acetate were purchased from Merck. De-ionised water was prepared by an Elix 10/Milli-Q Gradient (Millipore, Billerica, MA, USA). Acetone- $d_{6}$, chloroform- $d$, methanol- $d_{4}$ and pyridine- $d_{5}$ for NMR measurements were purchased from Merck. MTT (3-[4,5-dimethylthiazol-2-yl]-2,5-diphenyltetrazolium bromide) and Dulbecco's modified Eagle's medium (DMEM) were purchased from Sigma Chemical Co. (St. Louis, MO, USA). FBS (Fetal bovine serum) and penicillin/streptomycin were purchased from Gibco-BRL (New York, NY, USA). The A549 cell line was purchased from the Food Industry Research and Development Institute (Hsinchu, Taiwan).

\subsection{Plant Material}

The leaves of E. oldhamii Maxim. as described in Flora of Taiwan [1] were purchased from the Jin-Shun Shen herbal garden (Nantou, Taiwan) in October 2012. The plant materials were also identified by Yuan-Shiun Chang, Department of Chinese Pharmaceutical Sciences and Chinese Medicine Resources, College of Pharmacy, China Medical University, Taichung, Taiwan. A plant specimen has been deposited in the Department of Chinese Pharmaceutical Sciences and Chinese Medicine Resources with voucher specimen number CMU-CMR-2013-EO-1.

\subsection{Extraction and Isolation}

The materials were totally dried in air under dark. Dried leaves of E. oldhamii Maxim. (10.0 kg) were cut into small pieces and soaked in $70 \mathrm{~L}$ of methanol for 7 days. The extraction was repeated three times. The extracts were combined and filtered through filter paper and concentrated under reduced pressure to give a dried extract $(801.0 \mathrm{~g})$. The dried extract was suspended in $\mathrm{H}_{2} \mathrm{O}(2 \mathrm{~L})$ and extracted with ethyl acetate $(2 \mathrm{~L}$, five times). The resulting ethyl acetate extract was concentrated to yield $302.5 \mathrm{~g}$ of a brown-green thick oil. The fraction was further purified on $2.2 \mathrm{~kg}$ of silica gel with particle size $0.063-0.200 \mathrm{~mm}$ on a column with $10 \mathrm{~cm}$ internal diameter and $50 \mathrm{~cm}$ length using a gradient of increasing polarity from total $n$-hexane to total ethyl acetate as mobile phase and was 
separated into 20 fractions. Fraction $1(0.52 \mathrm{~g})$ was isolated from the $100 \% n$-hexane mobile phase. Fraction $2(12.28 \mathrm{~g})$ was isolated from the 1:19 $n$-hexane/ethyl acetate mobile phase. Fraction $3(18.95 \mathrm{~g})$ was isolated from the 1:9 $n$-hexane/ethyl acetate mobile phase. Fractions 4 (10.28 g), 5 (11.41 g), $6(10.55 \mathrm{~g}), 7(15.20 \mathrm{~g})$ and $8(8.85 \mathrm{~g})$ were isolated from the $8: 2 n$-hexane/ethyl acetate mobile phase. Fractions $9(16.69 \mathrm{~g})$ and $10(13.44 \mathrm{~g})$ were isolated from the 7:3 $n$-hexane/ethyl acetate mobile phase. Fractions $11(19.18 \mathrm{~g}), 12(11.74 \mathrm{~g}), 13(7.84 \mathrm{~g})$ and $14(11.40 \mathrm{~g})$ were isolated from the 5:5 $n$-hexane/ethyl acetate mobile phase. Fractions $15(6.02 \mathrm{~g}), 16(14.38 \mathrm{~g})$ and $17(6.01 \mathrm{~g})$ were isolated from the 3:7 $n$-hexane/ethyl acetate mobile phase. Fractions 18 (19.91 g), 19 (12.57 g) and 20 (6.13 g) were isolated from the $100 \%$ ethyl acetate mobile phase. The ethyl acetate extract remaining on the column was eluted by methanol $(28.92 \mathrm{~g})$. The weight of the 20 fractions and the fraction which was eluted by methanol was $261.94 \mathrm{~g}$, so the recovery rate was $86.59 \%$.

Twenty five compounds were isolated in fractions 8,14 and 15 respectively. Compounds 1 (645.0 mg), 2 (32.1 mg), 3 (22.5 mg), 5 (655.2 mg), 6 (52.0 mg), 7 (15.3 mg), 9 (10.2 mg), 10 (8.5 mg), 11 $(11.5 \mathrm{mg}), \mathbf{1 2}(14.0 \mathrm{mg})$ and $\mathbf{1 3}(12.8 \mathrm{mg})$ were isolated from fraction 8. Compounds $\mathbf{4}(8.8 \mathrm{mg}), \mathbf{8}$ (13.7 mg), 14 (22.6 mg), 15 (2.7 mg), 16 (11.7 mg), 19 (11.8 mg), 20 (22.0 mg), 21 (45.2 mg), 22 $(8.2 \mathrm{mg}), \mathbf{2 3}(5.8 \mathrm{mg}), \mathbf{2 4}(14.0 \mathrm{mg})$ and $\mathbf{2 5}(8.3 \mathrm{mg})$ were isolated from fraction 14. Compounds 17 (32.8 $\mathrm{mg})$, and $18(10.3 \mathrm{mg})$ were isolated from fraction 15 . The isolation procedure of these 25 compounds was described below.

Fraction $8(8.85 \mathrm{~g})$ was washed with $n$-hexane and acetone to obtain a mixture of compounds $\mathbf{1}$ and 5 (total weight equivalent to $1,306.5 \mathrm{mg}$ ). These two compounds were separated by semi-preparative RP-HPLC (acetonitrile/0.1\% ammonium acetate solution $=9: 1, \mathrm{v} / \mathrm{v}$ ) to afford pure compounds 1 and 5. The remaining fraction $8(8.14 \mathrm{~g})$ was separated by silica gel column chromatography ( $n$-hexane/ethyl acetate $=8: 2, \mathrm{v} / \mathrm{v}$ ) to give four subfractions (subfraction 8-1 to 8-4). Compounds 2, 3, 6 and 7 were obtained from subfraction $8-1$ through semi-preparative NP-HPLC ( $n$-hexane/acetone $=$ 8:2, v/v). Compounds 9 and $\mathbf{1 0}$ were obtained from subfraction 8-2 through semi-preparative NP-HPLC $(n$-hexane/acetone $=8: 2, \mathrm{v} / \mathrm{v})$ and semi-preparative RP-HPLC $($ acetonitrile $/$ water $=19: 1, \mathrm{v} / \mathrm{v})$. Compound 11 was obtained from subfraction $8-3$ through semi-preparative NP-HPLC ( $n$-hexane/acetone $=8: 2$, v/v). Compounds 12 and 13 were obtained from subfraction 8-4 through semi-preparative NP-HPLC $(n$-hexane/acetone $=8: 2, \mathrm{v} / \mathrm{v})$.

Fraction $14(11.40 \mathrm{~g})$ was separated by Sephadex LH 20 column chromatography (chloroform $/$ methanol $=3: 7, \mathrm{v} / \mathrm{v}$ ) to give six subfractions (subfraction 14-1 to 14-6). Compounds 4, 8, 23 and 25 were obtained from subfraction 14-2 through semi-preparative NP-HPLC $(n$-hexane/acetone $=1: 1, \mathrm{v} / \mathrm{v})$ and semi-preparative RP-HPLC (acetonitrile/water $=8: 2, \mathrm{v} / \mathrm{v})$. Compound 19 was obtained from subfraction 14-2 through semi-preparative NP-HPLC $(n$-hexane/acetone $=1: 1, \mathrm{v} / \mathrm{v}$ ) and NP-HPLC ( $n$-hexane/ethyl acetate $=1: 1, \mathrm{v} / \mathrm{v}$ ). Compound 22 was obtained from subfraction 14-3 through semi-preparative NP-HPLC ( $n$-hexane/acetone $=1: 1, \mathrm{v} / \mathrm{v})$. Compound 24 was obtained from subfraction 14-3 through semi-preparative NP-HPLC ( $n$-hexane/acetone $=1: 1, \mathrm{v} / \mathrm{v}$ ) and semi-preparative RP-HPLC ( $n$-hexane/ethyl acetate $=1: 1, \mathrm{v} / \mathrm{v}$ ). Compound $\mathbf{2 0}$ was obtained from subfraction 14-4 through semi-preparative NP-HPLC ( $n$-hexane/acetone $=3: 2, \mathrm{v} / \mathrm{v}$ ) and NP-HPLC $(n$-hexane/ethyl acetate $=52: 48, \mathrm{v} / \mathrm{v})$. Compounds 14, 15, and 21 were obtained from subfraction 14-5 through semi-preparative NP-HPLC ( $n$-hexane/acetone $=8: 2, \mathrm{v} / \mathrm{v}$ ). Compound 16 was obtained from subfraction 14-5 through semi-preparative NP-HPLC ( $n$-hexane/acetone $=8: 2, \mathrm{v} / \mathrm{v})$ and semi-preparative 
NP-HPLC ( $n$-hexane/ethyl acetate $=2: 3, \mathrm{v} / \mathrm{v})$. Fraction $15(6.02 \mathrm{~g})$ was separated by Sephadex LH 20 column chromatography (chloroform/methanol $=3: 7$, v/v) to give four subfractions (subfraction 15-1 to 15-4). Compounds $\mathbf{1 7}$ and $\mathbf{1 8}$ were obtained from subfraction 15-3 through semi-preparative NP-HPLC $(n$-hexane/acetone $=2: 3, \mathrm{v} / \mathrm{v})$ and semi-preparative NP-HPLC $(n$-hexane/acetone $=1: 2, \mathrm{v} / \mathrm{v})$.

\subsection{Spectroscopic Data}

Oleanolic acid (1). White powder, ${ }^{1} \mathrm{H}-\mathrm{NMR}\left(500 \mathrm{MHz}, \mathrm{C}_{5} \mathrm{D}_{5} \mathrm{~N}\right): \delta 5.52(1 \mathrm{H}, b r s, \mathrm{H}-12), \delta 3.46(1 \mathrm{H}$, $d d, J=10.5,5.5 \mathrm{~Hz}, \mathrm{H}-3), \delta 3.33(1 \mathrm{H}, d d, J=13.8,3.7 \mathrm{~Hz}, \mathrm{H}-18), \delta 1.30(3 \mathrm{H}, s), \delta 1.26(3 \mathrm{H}, s)$, $\delta 1.04(6 \mathrm{H}, s), \delta 1.03(3 \mathrm{H}, s), \delta 0.97(3 \mathrm{H}, s), \delta 0.91(3 \mathrm{H}, s), \delta 0.88(1 \mathrm{H}, d, J=12.0 \mathrm{~Hz}, \mathrm{H}-5)$. The above data were identical to the literature data [32].

3-O-(Z)-Coumaroyl oleanolic acid (2). White powder, ${ }^{1} \mathrm{H}-\mathrm{NMR}\left(500 \mathrm{MHz}, \mathrm{C}_{5} \mathrm{D}_{5} \mathrm{~N}\right): \delta 8.12(2 \mathrm{H}, d$, $J=8.6 \mathrm{~Hz}, \mathrm{H}-5^{\prime}$ and $\left.\mathrm{H}-9^{\prime}\right), \delta 7.21\left(2 \mathrm{H}, d, J=8.6 \mathrm{~Hz}, \mathrm{H}-6^{\prime}\right.$ and $\left.\mathrm{H}-8^{\prime}\right), \delta 7.01\left(1 \mathrm{H}, d, J=12.9 \mathrm{~Hz}, \mathrm{H}-3^{\prime}\right), \delta$ $6.05\left(1 \mathrm{H}, d, J=12.9 \mathrm{~Hz}, \mathrm{H}-2^{\prime}\right), \delta 5.50(1 \mathrm{H}, b r s, \mathrm{H}-12), \delta 4.82(1 \mathrm{H}, d d, J=11.8,4.6 \mathrm{~Hz}, \mathrm{H}-3)$, $\delta 1.28(3 \mathrm{H}, s), \delta 1.03(3 \mathrm{H}, s), \delta 1.00(3 \mathrm{H}, s), \delta 0.98(3 \mathrm{H}, s), \delta 0.97(3 \mathrm{H}, s), \delta 0.90(3 \mathrm{H}, s), \delta 0.85(3 \mathrm{H}, s)$. The above data were identical to the literature data [33].

3-O-(E)-Coumaroyl oleanolic acid (3). White powder, ${ }^{1} \mathrm{H}-\mathrm{NMR}\left(500 \mathrm{MHz}, \mathrm{C}_{5} \mathrm{D}_{5} \mathrm{~N}\right): \delta 8.03(1 \mathrm{H}, d$, $\left.J=15.9 \mathrm{~Hz}, \mathrm{H}-3^{\prime}\right), \delta 7.68\left(2 \mathrm{H}, d, J=8.6 \mathrm{~Hz}, \mathrm{H}-5^{\prime}\right.$ and $\left.\mathrm{H}-9^{\prime}\right), \delta 7.19\left(2 \mathrm{H}, d, J=8.6 \mathrm{~Hz}, \mathrm{H}-6{ }^{\prime}\right.$ and $\left.\mathrm{H}^{-8}\right), \delta 6.71\left(1 \mathrm{H}, d, J=15.9 \mathrm{~Hz}, \mathrm{H}-2^{\prime}\right), \delta 5.49(1 \mathrm{H}, b r s, \mathrm{H}-12), \delta 4.90(1 \mathrm{H}, b r d, J=9.8 \mathrm{~Hz}, \mathrm{H}-3)$, $\delta 1.29(3 \mathrm{H}, s), \delta 1.03(3 \mathrm{H}, s), \delta 1.02(3 \mathrm{H}, s), \delta 0.98(6 \mathrm{H}, s), \delta 0.97(3 \mathrm{H}, s), \delta 0.87(3 \mathrm{H}, s)$. The above data were identical to the literature data [33].

3-O-Caffeoyl oleanolic acid (4). White powder, ${ }^{1} \mathrm{H}-\mathrm{NMR}\left(500 \mathrm{MHz}, \mathrm{CD}_{3} \mathrm{OD}\right): \delta 7.53(1 \mathrm{H}, d, J=15.8$ Hz, H-3'), $\delta 7.03\left(1 \mathrm{H}, s, \mathrm{H}-5^{\prime}\right), \delta 6.93\left(1 \mathrm{H}, d, J=8.1 \mathrm{~Hz}, \mathrm{H}-9^{\prime}\right), \delta 6.77\left(1 \mathrm{H}, d, J=8.1 \mathrm{~Hz}, \mathrm{H}-8^{\prime}\right), \delta 6.23$ $\left(1 \mathrm{H}, d, J=15.8 \mathrm{~Hz}, \mathrm{H}-2^{\prime}\right), \delta 5.24(1 \mathrm{H}, b r s, \mathrm{H}-12), \delta 4.56(1 \mathrm{H}, b r d, J=10.0 \mathrm{~Hz}, \mathrm{H}-3), \delta 1.17(3 \mathrm{H}, s), \delta$ $1.00(3 \mathrm{H}, s), \delta 0.96(3 \mathrm{H}, s), \delta 0.94(3 \mathrm{H}, s), \delta 0.90(6 \mathrm{H}, s), \delta 0.83(3 \mathrm{H}, s)$. The above data were identical to the literature data [34].

Ursolic acid (5). White powder, ${ }^{1} \mathrm{H}-\mathrm{NMR}\left(500 \mathrm{MHz}, \mathrm{C}_{5} \mathrm{D}_{5} \mathrm{~N}\right): \delta 5.49(1 \mathrm{H}, b r s, \mathrm{H}-12), \delta 3.45(1 \mathrm{H}, d d$, $J=9.7,6.4 \mathrm{~Hz}, \mathrm{H}-3), \delta 2.63(1 \mathrm{H}, d, J=11.4 \mathrm{~Hz}, \mathrm{H}-18), 1.24(3 \mathrm{H}, s), 1.23(3 \mathrm{H}, s), 1.05(3 \mathrm{H}, s), \delta 1.02$ $(3 \mathrm{H}, s), \delta 1.00(1 \mathrm{H}, d, J=6.5 \mathrm{~Hz}), \delta 0.96(1 \mathrm{H}, d, J=6.2 \mathrm{~Hz}), \delta 0.89(3 \mathrm{H}, s), \delta 0.86(1 \mathrm{H}, d, J=11.5 \mathrm{~Hz}$, $\mathrm{H}-5)$. The above data were identical to the literature data [32].

3-O-(Z)-coumaroyl ursolic acid (6) White powder, ${ }^{1} \mathrm{H}-\mathrm{NMR}\left(500 \mathrm{MHz}, \mathrm{CDCl}_{3}\right): \delta 7.59(2 \mathrm{H}, d, J=8.1 \mathrm{~Hz}$, H-5' and H-9'), $\delta 6.76\left(2 \mathrm{H}, d, J=8.1 \mathrm{~Hz}, \mathrm{H}-6^{\prime}\right.$ and $\left.\mathrm{H}^{\prime} 8^{\prime}\right), \delta 6.84\left(1 \mathrm{H}, d, J=12.8 \mathrm{~Hz}, \mathrm{H}-3^{\prime}\right), \delta 5.83(1 \mathrm{H}$, $\left.d, J=12.8 \mathrm{~Hz}, \mathrm{H}-2^{\prime}\right), \delta 5.23(1 \mathrm{H}, b r s, \mathrm{H}-12), \delta 4.56(1 \mathrm{H}, b r d, J=9.3 \mathrm{~Hz}, \mathrm{H}-3), \delta 1.08(3 \mathrm{H}, s)$, $\delta 0.95(6 \mathrm{H}, s), \delta 0.87(3 \mathrm{H}, s), \delta 0.86(3 \mathrm{H}, s), \delta 0.82(3 \mathrm{H}, s), \delta 0.76(3 \mathrm{H}, s)$. The above data were identical to the literature data [35].

3-O-(E)-Coumaroyl ursolic acid (7). White powder, ${ }^{1} \mathrm{H}-\mathrm{NMR}\left(500 \mathrm{MHz}, \mathrm{CD}_{3} \mathrm{OD}\right): \delta 7.62(1 \mathrm{H}, d$, $\left.J=15.9 \mathrm{~Hz}, \mathrm{H}-3^{\prime}\right), \delta 7.45\left(2 \mathrm{H}, d, J=8.6 \mathrm{~Hz}, \mathrm{H}-5^{\prime}\right.$ and $\left.\mathrm{H}-9^{\prime}\right), \delta 6.80\left(2 \mathrm{H}, d, J=8.6 \mathrm{~Hz}, \mathrm{H}-6{ }^{\prime}\right.$ and $\left.\mathrm{H}^{-} 8^{\prime}\right), \delta 6.38\left(1 \mathrm{H}, d, J=15.9 \mathrm{~Hz}, \mathrm{H}-2^{\prime}\right), \delta 5.24(1 \mathrm{H}, b r s, \mathrm{H}-12), \delta 4.63(1 \mathrm{H}, b r d, J=9.9 \mathrm{~Hz}, \mathrm{H}-3)$, 
$\delta 1.14(3 \mathrm{H}, s), \delta 1.06(3 \mathrm{H}, s), \delta 0.97(3 \mathrm{H}, s), \delta 0.94(3 \mathrm{H}, s), \delta 0.89(3 \mathrm{H}, s), \delta 0.88(3 \mathrm{H}, s), \delta 0.86(3 \mathrm{H}, s)$. The above data were identical to the literature data [35].

3-O-Caffeoyl ursolic acid (8). White powder, ${ }^{1} \mathrm{H}-\mathrm{NMR}\left(500 \mathrm{MHz}, \mathrm{CD}_{3} \mathrm{OD}\right): \delta 7.53(1 \mathrm{H}, d, J=15.9$ Hz, H-3'), $\delta 7.16\left(1 \mathrm{H}, s, \mathrm{H}-5^{\prime}\right), \delta 7.03\left(1 \mathrm{H}, d, J=8.2 \mathrm{~Hz}, \mathrm{H}-9{ }^{\prime}\right), \delta 6.85\left(1 \mathrm{H}, d, J=8.2 \mathrm{~Hz}, \mathrm{H}-8^{\prime}\right), \delta 6.29$ $\left(1 \mathrm{H}, d, J=15.9 \mathrm{~Hz}, \mathrm{H}-2^{\prime}\right), \delta 5.25(1 \mathrm{H}, b r s, \mathrm{H}-12), \delta 4.75(1 \mathrm{H}, d d, J=11.4,4.5 \mathrm{~Hz}, \mathrm{H}-3), \delta 1.20(3 \mathrm{H}, s)$, $\delta 1.00(3 \mathrm{H}, s), \delta 0.95(3 \mathrm{H}, s), \delta 0.94(3 \mathrm{H}, s), \delta 0.92(3 \mathrm{H}, s), \delta 0.90(3 \mathrm{H}, s), \delta 0.82(3 \mathrm{H}, s)$. The above data were identical to the literature data [36].

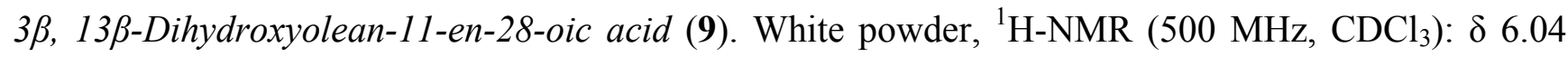
$(1 \mathrm{H}, d, J=10.2 \mathrm{~Hz}, \mathrm{H}-12), \delta 5.41(1 \mathrm{H}, d d, J=10.2,2.4 \mathrm{~Hz}, \mathrm{H}-11), \delta 3.22(1 \mathrm{H}, d d, J=11.4,4.3 \mathrm{~Hz}$, $\mathrm{H}-3), \delta 1.25(3 \mathrm{H}, s), \delta 1.06(3 \mathrm{H}, s), \delta 0.98(3 \mathrm{H}, s), \delta 0.97(3 \mathrm{H}, s), \delta 0.91(3 \mathrm{H}, s), \delta 0.88(3 \mathrm{H}, s), \delta 0.78$ $(3 \mathrm{H}, s)$. The above data were identical to the literature data [37].

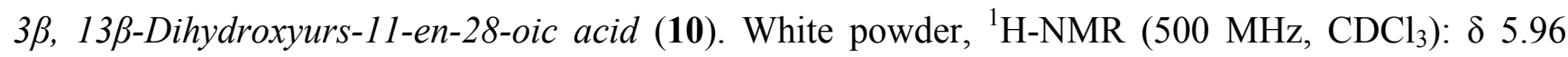
$(1 \mathrm{H}, d, J=10.2 \mathrm{~Hz}, \mathrm{H}-12), \delta 5.25(1 \mathrm{H}, b r d, J=10.2, \mathrm{H}-11), \delta 3.22(1 \mathrm{H}, d d, J=11.6,4.6 \mathrm{~Hz}, \mathrm{H}-3), \delta$ $1.16(3 \mathrm{H}, s), \delta 1.00(3 \mathrm{H}, d, J=6.3), \delta 0.99(3 \mathrm{H}, s), \delta 0.94(3 \mathrm{H}, s), \delta 0.94(3 \mathrm{H}, d, J=6.3), \delta 0.91(3 \mathrm{H}$, $s), \delta 0.78(3 \mathrm{H}, s)$. The above data were identical to the literature data [38].

Uvaol (11). White powder, ${ }^{1} \mathrm{H}-\mathrm{NMR}\left(500 \mathrm{MHz}, \mathrm{CDCl}_{3}\right): \delta 5.12(1 \mathrm{H}, t, J=3.5 \mathrm{~Hz}, \mathrm{H}-12), \delta 3.54(1 \mathrm{H}$, $d, J=11.0 \mathrm{~Hz}, \mathrm{H}-28 \beta), \delta 3.20(1 \mathrm{H}, d d, J=11.2,5.0 \mathrm{~Hz}, \mathrm{H}-3), \delta 3.18(1 \mathrm{H}, d, J=11.0 \mathrm{~Hz}, \mathrm{H}-28 \alpha)$, $\delta 1.09(3 \mathrm{H}, s), \delta 0.99(3 \mathrm{H}, s), \delta 0.94(3 \mathrm{H}, s), \delta 0.93(3 \mathrm{H}, d, J=5.8 \mathrm{~Hz}), \delta 0.80(3 \mathrm{H}, d, J=5.8 \mathrm{~Hz})$, $\delta 0.78(3 \mathrm{H}, s), \delta 0.72(3 \mathrm{H}, d, J=11.2 \mathrm{~Hz}, \mathrm{H}-5)$. The above data were identical to the literature data [39].

Betulin (12). White powder, ${ }^{1} \mathrm{H}-\mathrm{NMR}\left(500 \mathrm{MHz}, \mathrm{CDCl}_{3}\right): \delta 4.68(1 \mathrm{H}, \mathrm{br} s, \mathrm{H}-29 \beta), \delta 4.58(1 \mathrm{H}, \mathrm{br} s$, $\mathrm{H}-29 \alpha), \delta 3.79(1 \mathrm{H}, d, J=10.8 \mathrm{~Hz}, \mathrm{H}-28 \beta), \delta 3.32(1 \mathrm{H}, d, J=10.8 \mathrm{~Hz}, \mathrm{H}-28 \alpha), \delta 3.20(1 \mathrm{H}, d d$, $J=11.4,4.6 \mathrm{~Hz}, \mathrm{H}-3), \delta 1.67(3 \mathrm{H}, s), \delta 1.01(6 \mathrm{H}, s), \delta 0.98(3 \mathrm{H}, s), \delta 0.96(3 \mathrm{H}, s), \delta 0.82(3 \mathrm{H}, s)$, $\delta 0.75(3 \mathrm{H}, s)$. The above data were identical to the literature data [40].

Lupeol (13). White powder, ${ }^{1} \mathrm{H}-\mathrm{NMR}\left(500 \mathrm{MHz}, \mathrm{CDCl}_{3}\right): \delta 4.68(1 \mathrm{H}, \mathrm{br} s, \mathrm{H}-29 \beta), \delta 4.56(1 \mathrm{H}, \mathrm{br} s$, $\mathrm{H}-29 \alpha), \delta 3.18(1 \mathrm{H}, d d, J=11.4,4.9 \mathrm{~Hz}, \mathrm{H}-3), \delta 1.68(3 \mathrm{H}, s), \delta 1.02(3 \mathrm{H}, s), \delta 0.96(3 \mathrm{H}, s), \delta 0.94(3 \mathrm{H}, s)$, $\delta 0.83(3 \mathrm{H}, s), \delta 0.79(3 \mathrm{H}, s), \delta 0.76(3 \mathrm{H}, s)$. The above data were identical to the literature data [41].

Kaempferol (14). Yellowish powder, ${ }^{1} \mathrm{H}-\mathrm{NMR}\left(500 \mathrm{MHz}, \mathrm{CD}_{3} \mathrm{OD}\right): \delta 8.06\left(2 \mathrm{H}, d, J=8.8 \mathrm{~Hz}, \mathrm{H}-2^{\prime}\right.$ and $\left.\mathrm{H}_{-} 6^{\prime}\right), \delta 6.88\left(2 \mathrm{H}, d, J=8.8 \mathrm{~Hz}, \mathrm{H}-3^{\prime}\right.$ and $\left.\mathrm{H}_{-} 5^{\prime}\right), \delta 6.37(1 \mathrm{H}, d, J=1.7 \mathrm{~Hz}, \mathrm{H}-8), \delta 6.16(1 \mathrm{H}, d$, $J=1.7 \mathrm{~Hz}, \mathrm{H}-6)$. The above data were identical to the literature data [42].

Aromadendrin (15). White powder, ${ }^{1} \mathrm{H}-\mathrm{NMR}\left(500 \mathrm{MHz}, \mathrm{CD}_{3} \mathrm{OD}\right): \delta 11.71(1 \mathrm{H}, b r s, \mathrm{OH}), \delta 7.41(2 \mathrm{H}$, $d, J=8.5 \mathrm{~Hz}, \mathrm{H}-2^{\prime}$ and H-6'), $\delta 6.89\left(2 \mathrm{H}, d, J=8.5 \mathrm{~Hz}, \mathrm{H}-3^{\prime}\right.$ and $\left.\mathrm{H}-5^{\prime}\right), \delta 5.99(1 \mathrm{H}, d, J=1.9 \mathrm{~Hz}$, $\mathrm{H}-8), \delta 5.94(1 \mathrm{H}, d, J=1.9 \mathrm{~Hz}, \mathrm{H}-6), \delta 5.08(1 \mathrm{H}, d, J=11.5 \mathrm{~Hz}, \mathrm{H}-2), \delta 5.65(1 \mathrm{H}, d, J=11.5 \mathrm{~Hz}$, $\mathrm{H}-3)$. The above data were identical to the literature data [43].

Epigallocatechin (16). White powder, ${ }^{1} \mathrm{H}-\mathrm{NMR}\left(500 \mathrm{MHz}, \mathrm{CD}_{3} \mathrm{OD}\right): \delta 8.17(1 \mathrm{H}, \mathrm{br} s, \mathrm{OH}), \delta 8.01$ $(1 \mathrm{H}, b r s, \mathrm{OH}), \delta 7.92(2 \mathrm{H}, b r s, \mathrm{OH}), \delta 6.57\left(2 \mathrm{H}, s, \mathrm{H}-3^{\prime}\right.$ and $\left.\mathrm{H}-5^{\prime}\right), \delta 6.01(1 \mathrm{H}, d, J=2.3 \mathrm{~Hz}, \mathrm{H}-6), \delta$ 
$5.91(1 \mathrm{H}, d, J=2.3 \mathrm{~Hz}, \mathrm{H}-8), \delta 4.81\left(1 \mathrm{H}, s, \mathrm{H}-2^{\prime}\right), \delta 4.18(1 \mathrm{H}, d, J=3.5 \mathrm{~Hz}, \mathrm{H}-3), \delta 3.52(1 \mathrm{H}, d, J=5.1$ $\mathrm{Hz}, \mathrm{OH}), \delta 2.74(1 \mathrm{H}, d d, J=16.5,4.6 \mathrm{~Hz}, \mathrm{H}-4 \alpha), \delta 2.71(1 \mathrm{H}, d d, J=16.5,3.4 \mathrm{~Hz}, \mathrm{H}-4 \beta)$. The above data were identical to the literature data [44].

cis-Tiliroside (17). Yellowish powder, ${ }^{1} \mathrm{H}-\mathrm{NMR}\left(500 \mathrm{MHz}, \mathrm{CD}_{3} \mathrm{COCD}_{3}\right): \delta 12.37(1 \mathrm{H}, \mathrm{br} s, \mathrm{OH})$, $\delta 8.09\left(2 \mathrm{H}, d, J=8.8 \mathrm{~Hz}, \mathrm{H}-22^{\prime}\right.$ and H-6'), $\delta 7.68$ (2H, $d, J=8.6 \mathrm{~Hz}, \mathrm{H}-2{ }^{\prime \prime}$ and H-6"'), $\delta 6.92(2 \mathrm{H}, d$, $J=8.8 \mathrm{~Hz}, \mathrm{H}-3^{\prime}$ and H-5'), $\delta 6.78\left(2 \mathrm{H}, d, J=8.6 \mathrm{~Hz}, \mathrm{H}-3^{\prime \prime \prime}\right.$ and H-5"'), $\delta 6.78(1 \mathrm{H}, d, J=12.9 \mathrm{~Hz}$, H-7"'), $\delta 6.47$ (1H, br s, H-8), $\delta 6.27$ (1H, br s, H-6), $\delta 5.62\left(1 \mathrm{H}, d, J=12.9 \mathrm{~Hz}, \mathrm{H}-8{ }^{\prime \prime}\right), \delta 5.17(1 \mathrm{H}, d$, $\left.J=7.3 \mathrm{~Hz}, \mathrm{H}-1^{\prime \prime}\right), \delta 4.28\left(1 \mathrm{H}, d d, J=11.9,1.8 \mathrm{~Hz}, \mathrm{H}-6 \beta^{\prime \prime}\right), \delta 4.17(1 \mathrm{H}, d d, J=11.9,6.0 \mathrm{~Hz}$, H-6a"), $\delta 3.55$ (1H, m, H-2"), $\delta 3.55$ (1H, m, H-5"), $\delta 3.49$ (1H, $t, J=8.9 \mathrm{~Hz}, \mathrm{H}-4 "), \delta 3.41$ (1H, $d$, $J=9.3 \mathrm{~Hz}, \mathrm{H}-3 ")$. The above data were identical to the literature data [45].

trans-Tiliroside (18). Yellowish powder, ${ }^{1} \mathrm{H}-\mathrm{NMR}\left(500 \mathrm{MHz}, \mathrm{CD}_{3} \mathrm{OD}\right): \delta 7.96\left(2 \mathrm{H}, d, J=8.8 \mathrm{~Hz}, \mathrm{H}-2^{\prime}\right.$ and H-6'), $\delta 7.38\left(1 \mathrm{H}, d, J=16.5 \mathrm{~Hz}, \mathrm{H}-7^{\prime \prime}\right), \delta 7.28\left(2 \mathrm{H}, d, J=8.5 \mathrm{~Hz}, \mathrm{H}-2^{\prime \prime \prime}\right.$ and H-6"'), $\delta 6.80(2 \mathrm{H}, d$, $J=8.8 \mathrm{~Hz}, \mathrm{H}-3^{\prime}$ and H-5'), $\delta 6.78\left(2 \mathrm{H}, d, J=8.5 \mathrm{~Hz}, \mathrm{H}-3^{\prime \prime}\right.$ and H-5"'), $\delta 6.27(1 \mathrm{H}, b r s$, $\mathrm{H}-8), \delta 6.11(1 \mathrm{H}, b r s, \mathrm{H}-6), \delta 6.05\left(1 \mathrm{H}, d, J=16.5 \mathrm{~Hz}, \mathrm{H}-8^{\prime \prime \prime}\right), \delta 5.23$ (1H, $\left.d, J=7.4 \mathrm{~Hz}, \mathrm{H}-1 "\right)$, $\delta 4.31\left(1 \mathrm{H}, d d, J=11.8,1.9 \mathrm{~Hz}, \mathrm{H}-6 \beta^{\prime \prime}\right), \delta 4.18\left(1 \mathrm{H}, d d, J=11.8,6.6 \mathrm{~Hz}, \mathrm{H}-6 \alpha^{\prime \prime}\right), \delta 3.55(1 \mathrm{H}, m$, H-5"), $\delta 3.48$ (1H, $m, \mathrm{H}-3 "), \delta 3.47$ (1H, $m, \mathrm{H}-2 "), \delta 3.46$ (1H, $m, \mathrm{H}-4 ")$. The above data were identical to the literature data [45].

Isoamericanol $B$ (19). Yellowish oil, ${ }^{1} \mathrm{H}-\mathrm{NMR}\left(500 \mathrm{MHz}, \mathrm{CD}_{3} \mathrm{COCD}_{3}\right): \delta 7.69(1 \mathrm{H}, s, \mathrm{OH}), \delta 7.06$ $(1 \mathrm{H}, d, J=1.6 \mathrm{~Hz}, \mathrm{H}-2), \delta 6.92(1 \mathrm{H}, d d, J=8.2,1.6 \mathrm{~Hz}, \mathrm{H}-6), \delta 6.87(1 \mathrm{H}, d, J=8.2 \mathrm{~Hz}, \mathrm{H}-5), \delta 6.87$ $\left(1 \mathrm{H}, d, J=1.8 \mathrm{~Hz}, \mathrm{H}-2^{\prime}\right), \delta 6.86\left(1 \mathrm{H}, d, J=8.2 \mathrm{~Hz}, \mathrm{H}-5^{\prime}\right), \delta 6.79\left(1 \mathrm{H}, d d, J=8.2,1.8 \mathrm{~Hz}, \mathrm{H}-6^{\prime}\right)$, $\delta 6.38\left(1 \mathrm{H}, d, J=11.8 \mathrm{~Hz}, \mathrm{H}-7^{\prime}\right), \delta 5.76\left(1 \mathrm{H}, m, \mathrm{H}-8^{\prime}\right), \delta 5.18(1 \mathrm{H}, d, J=2.6 \mathrm{~Hz}, \mathrm{H}-7), \delta 4.60(1 \mathrm{H}, d$, $J=6.6,2.6 \mathrm{~Hz}, \mathrm{H}-8), \delta 4.38(2 \mathrm{H}, d, J=5.0 \mathrm{~Hz}, \mathrm{H}-9), \delta 3.83\left(3 \mathrm{H}, s, \mathrm{OCH}_{3}\right), \delta 1.68(1 \mathrm{H}, b r s, \mathrm{OH})$, $\delta 1.08(3 \mathrm{H}, d, J=6.6 \mathrm{~Hz}, \mathrm{H}-9)$. The above data were identical to the literature data [46].

trans-p-Coumaric acid (20) White powder, ${ }^{1} \mathrm{H}-\mathrm{NMR}\left(500 \mathrm{MHz}, \mathrm{CD}_{3} \mathrm{OD}\right): \delta 7.58(1 \mathrm{H}, d, J=15.9 \mathrm{~Hz}$, $\mathrm{H}-7), \delta 7.43(2 \mathrm{H}, d, J=8.6 \mathrm{~Hz}, \mathrm{H}-2$ and H-6), $\delta 6.79(2 \mathrm{H}, d, J=8.6 \mathrm{~Hz}, \mathrm{H}-3$ and H-5), $\delta 6.27(1 \mathrm{H}, d$, $J=15.9 \mathrm{~Hz}, \mathrm{H}-8)$. The above data were identical to the literature data [47].

Protocatechuic acid (21) White powder, ${ }^{1} \mathrm{H}-\mathrm{NMR}\left(500 \mathrm{MHz}, \mathrm{CD}_{3} \mathrm{OD}\right): \delta 7.52(1 \mathrm{H}, d, J=1.9 \mathrm{~Hz}$, $\mathrm{H}-2), \delta 7.47(1 \mathrm{H}, d d, J=8.3,1.9 \mathrm{~Hz}, \mathrm{H}-6), \delta 6.89(1 \mathrm{H}, d, J=1.9 \mathrm{~Hz}, \mathrm{H}-5)$. The above data were identical to the literature data [48].

Salicylic acid (22) White powder, ${ }^{1} \mathrm{H}-\mathrm{NMR}\left(500 \mathrm{MHz}, \mathrm{CD}_{3} \mathrm{COCD}_{3}\right): \delta 7.84(1 \mathrm{H}, d, J=7.8 \mathrm{~Hz}, \mathrm{H}-6)$, $\delta 7.52(1 \mathrm{H}, t, J=7.8 \mathrm{~Hz}, \mathrm{H}-4), \delta 7.89(1 \mathrm{H}, d, J=8.3 \mathrm{~Hz}, \mathrm{H}-3), \delta 6.87(1 \mathrm{H}, t, J=8.5 \mathrm{~Hz}, \mathrm{H}-5)$. The above data were identical to the literature data [49].

trans-Ferulic acid (23). White powder, ${ }^{1} \mathrm{H}-\mathrm{NMR}\left(500 \mathrm{MHz}, \mathrm{CD}_{3} \mathrm{OD}\right): \delta 7.58(1 \mathrm{H}, d, J=15.9 \mathrm{~Hz}, \mathrm{H}-7), \delta$ $7.17(1 \mathrm{H}, b r s, \mathrm{H}-2), \delta 7.05(1 \mathrm{H}, b r d, J=8.1 \mathrm{~Hz}, \mathrm{H}-6), \delta 6.80(1 \mathrm{H}, d, J=8.1 \mathrm{~Hz}, \mathrm{H}-5), \delta 6.30(1 \mathrm{H}, d$, $J=15.9 \mathrm{~Hz}, \mathrm{H}-8), \delta 3.88\left(3 \mathrm{H}, s, \mathrm{OCH}_{3}\right)$. The above data were identical to the literature data [50]. 
Syringic acid (24). White powder, ${ }^{1} \mathrm{H}-\mathrm{NMR}\left(500 \mathrm{MHz}, \mathrm{CD}_{3} \mathrm{OD}\right): \delta 7.31$ (2H, s, H-2 and H-6), $\delta 3.87$ $\left(6 \mathrm{H}, s, \mathrm{OCH}_{3}\right)$. The above data were identical to the literature data [51].

3-O-Methylgallic acid (25) White powder, ${ }^{1} \mathrm{H}-\mathrm{NMR}$ (500 MHz, $\left.\mathrm{CD}_{3} \mathrm{OD}\right): \delta 7.17$ (2H, s, H-2 and H-6), $\delta 3.86\left(3 \mathrm{H}, s, \mathrm{OCH}_{3}\right)$. The above data were identical to the literature data [52].

\subsection{Cytotoxicity Assays}

\subsubsection{Cell Culture}

Cytotoxicity was measured using the MTT assay [57]. A549 (human lung adenocarcinoma) cell line was maintained in DMEM supplemented with 10\% fetal bovine serum (FBS), 100 units $/ \mathrm{mL}$ penicillin and $100 \mu \mathrm{g} / \mathrm{mL}$ streptomycin (Gibco-BRL). The cells were incubated in $5 \% \mathrm{CO}_{2}$ humidified at $37^{\circ} \mathrm{C}$ for growth.

\subsubsection{Evaluation of Cell Proliferation by MTT Assay}

The number of viable A549 cells after GA treatment was evaluated by the MTT (3-[4,5-methylthiazol-2-yl]-2,5-diphenyl-tetrazolium bromide) assay. In brief, A549 cells $\left(3 \times 10^{4}\right.$ cells/well) were seeded in a 96-well plate and kept overnight for attachment. The next day the medium was replaced with fresh medium with various concentrations of EOM extract, indicated fraction (EOE, EOB, and EOW), cisplatin (positive control) and 25 compounds which were isolated from the leaves of E. oldhamii Maxim. (5-500 $\mu \mathrm{g} / \mathrm{mL})$ and cells were allowed to grow for 24 and $48 \mathrm{~h}$. Four hours before completion of incubation, $10 \mu \mathrm{L}$ of MTT $(10 \mathrm{mg} / \mathrm{mL})$ was added in each well. After completing the incubation, $100 \mu \mathrm{L}$ of solubilization buffer $(10 \%$ SDS with $0.01 \mathrm{~N} \mathrm{HCl})$ was added to each well and incubated overnight at room temperature. The optical density (OD) was measured at 450 $\mathrm{nm}$ using a multiscan microplate reader. Data were showed means $\pm \mathrm{SD}$ from three independent experiments, $50 \%$ cytotoxic concentration $\left(\mathrm{CC}_{50}\right)$ yielding $50 \%$ toxic effect was determined via a computer program (provided by John Spouge, National Center for Biotechnology Information, National Institutes of Health).

\section{Conclusions}

Twenty five compounds were isolated in the present study, including thirteen triterpenoids, five flavonoids, one lignanoid and six benzanoids compounds. Furthermore, twenty one of these compounds, including compounds 1, 4, 5 and 8-25 were obtained from E. oldhamii Maxim. for the first time. Furthermore, we evaluated the inhibitory activity of these compounds against the growth of non-small cell lung cancer A549 cells by MTT assay in this study. Eleven of these 25 compounds, including compounds 2-4, 6-10, 13, 18 and 19 were investigated by the MTT assay for the first time regarding their cytotoxic activity in non-small cell lung cancer A549 cells. The result indicated that the five compounds, including four triterpenoids (compounds 2, 4, 6 and 8) and one flavanoid (compound 18) present in the crude extract exhibited good cytotoxic activity against the A549 cell line with $\mathrm{CC}_{50}$ values lower than $20 \mu \mathrm{g} / \mathrm{mL}$. Furthermore, in present study these five compounds exhibited better cytotoxic activity compared to cisplatin (positive control, Table 4). 
Regarding the structure-activity relationships of the triterpenoid compounds 2, 4, 6 and $\mathbf{8}$, a 3-O-linkage to the cis-coumaroyl and caffeoyl moieties of oleanolic acid and ursolic acid were two active functional groups that provided better cytotoxic activity against the growth of the non-small cell lung cancer A549 cells according to the MTT assays. In addition, regarding the structure-activity relationships of the flavanoid compound 18, a 3-O-linkage to D-glucose and a 6"-O-linkage to cis-coumaroyl were the active functional groups providing better cytotoxic activity against the growth of non-small cell lung cancer A549 cells in the MTT assay. These five compounds 2, 4, 6, 8 and 18 may lead to the development of anti-lung cancer drugs although further studies should be performed to reveal the mechanisms of action of the active compounds found in leaves of E. oldhamii Maxim.

\section{Acknowledgments}

This work was kindly supported by a grant from the CMU under the Aim for Top University Plan of the Ministry of Education, Taiwan and Taiwan Department of Health Clincical Trial and Research Center of Excellence (DOH-102-TD-B-111-004).

\section{Author Contributions}

Chi-Ren Liao, Chang Syun Yang and Ching-Ying Wang carried out the work. Yu-Ling Ho and Cheng-Wen Lin wrote the paper. Yueh-Hsiung Kuo helped structure elucidation work. Yuan-Shiun Chang coordinated the research team. All authors had read and approved the final manuscript.

\section{Conflicts of Interest}

The authors declare no conflict of interest.

\section{References}

1. Huang, T.S. Elaeagnaceae. In Flora of Taiwan, 2nd ed.; Editorial Committee of the Flora of Taiwan: Taipei, Taiwan, 1998; Volume 3, pp. 753-759.

2. Ahmad, S.D.; Sabir, M.S.; Juma, M.; Asad, H.S. Morphological and biochemical variations in Elaeagnus umbellata Thunb. From mountains of Pakistan. Acta Bot. Croat. 2005, 64, 121-128.

3. Yuebin, G.; Liu, J.; Su, D. In vivo evaluation of the anti-asthmatic, antitussive and expectorant activities of extract and fractions from Elaeagnus pungens leaf. J. Ethnopharmacol. 2009, 126, $538-542$.

4. Ahmadiani, A.; Hosseiny, J.; Semnanian, S.; Javan, M.; Saeedi, F.; Kamalinejad, M.; Saremi, S. Antinociceptive and anti-inflammatory effects of Elaeagnus angustifolia fruit extract. J. Ethnopharmacol. 2000, 72, 287-292.

5. Mehrabani Natanzi, M.; Pasalar, P.; Kamalinejad, M.; Dehpour, A.R.; Tavangar, S.M.; Sharifi, R.; Ghanadian, N.; Rahimi-Balaei, M.; Gerayesh-Nejad, S. Effect of aqueous extract of Elaeagnus angustifolia fruit on experimental cutaneous wound healing in rats. Acta Med. Iran. 2012, 50, 589-596. 
6. Lee, Y.S.; Chang, Z.Q.; Oh, B.C.; Park, S.C.; Shin, S.R.; Kim, N.W. Antioxidant activity, anti-inflammatory activity, and whitening effects of extracts of Elaeagnus multiflora Thunb. J. Med. Food 2007, 10, 126-133.

7. Talaei-Khozani, T.; Vojdani, Z.; Dehghani, F.; Heidari, E.; Kharazinejad, E.; Panjehshahin, M.R. Toxic effects of Elaeagnus angustifolia fruit extract on chondrogenesis and osteogenesis in mouse limb buds. Tokai J. Exp. Clin. Med. 2011, 36, 63-70.

8. Wang, S.Y.; Bowman, L.; Ding, M. Variations in free radical scavenging capacity and antiproliferative activity among different genotypes of autumn olive (Elaeagnus umbellata). Planta Med. 2007, 73, 468-477.

9. Wu, Y.B.; Gu, Y.; Ouyang, M.A. Water-soluble constituents from the bark of Elaeagnus pungens and their cytotoxic activities. J. Asian Nat. Prod. Res. 2010, 12, 278-285.

10. Li, L.H.; Baek, I.K.; Kim, J.H.; Kang, K.H.; Koh, Y.S.; Jung, Y.D.; Cho, C.K.; Choi, S.Y.; Shin, B.A. Methanol extract of Elaeagnus glabra, a Korean medicinal plant, inhibits HT1080 tumor cell invasion. Oncol. Rep. 2009, 21, 559-563.

11. Yan, M.C.; Liu, Y.; Chen, H.; Ke, Y.; Xu, Q.C.; Cheng, M.S. Synthesis and antitumor activity of two natural $\mathrm{N}$-acetylglucosamine-bearing triterpenoid saponins: Lotoidoside $\mathrm{D}$ and $\mathrm{E}$. Bioorg. Med. Chem. Lett. 2006, 16, 4200-4204.

12. Liu, Q.; Liu, H.; Zhang, L.; Guo, T.; Wang, P.; Geng, M.; Li, Y. Synthesis and antitumor activities of naturally occurring oleanolic acid triterpenoid saponins and their derivatives. Eur. $J$. Med. Chem. 2013, 64, 1-15.

13. Tu, J.; Sun, H.X.; Ye, Y.P. Immunomodulatory and antitumor activity of triterpenoid fractions from the rhizomes of Astilbe chinensis. J. Ethnopharmacol. 2008, 119, 266-271.

14. Park, K.I.; Park, H.S.; Nagappan, A.; Hong, G.E.; Lee do, H.; Kang, S.R.; Kim, J.A.; Zhang, J.; Kim, E.H.; Lee, W.S.; et al. Induction of the cell cycle arrest and apoptosis by flavonoids isolated from Korean Citrus aurantium L. in non-small cell lung cancer cells. Food Chem. 2012, 135, $2728-2735$.

15. Hung, J.Y.; Hsu, Y.L.; Ko, Y.C.; Tsai, Y.M.; Yang, C.J.; Huang, M.S.; Kuo, P.L. Didymin, a dietary flavonoid glycoside from citrus fruits, induces Fas-mediated apoptotic pathway in human non-small cell lung cancer cells in vitro and in vivo. Lung Cancer 2010, 68, 366-374.

16. Leung, H.W.; Lin, C.J.; Hour, M.J.; Yang, W.H.; Wang, M.Y.; Lee, H.Z. Kaempferol induces apoptosis in human lung non-small carcinoma cells accompanied by an induction of antioxidant enzymes. Food Chem. Toxicol. 2007, 45, 2005-2013.

17. Peuhu, E.; Paul, P.; Remes, M.; Holmbom, T.; Eklund, P.; Sjöholm, R.; Eriksson, J.E. The antitumor lignan Nortrachelogenin sensitizes prostate cancer cells to TRAIL-induced cell death by inhibition of the Akt pathway and growth factor signaling. Biochem. Pharmacol. 2013, $86,571-583$.

18. Vijayakurup, V.; Spatafora, C.; Tringali, C.; Jayakrishnan, P.C.; Srinivas, P.; Gopala, S. Phenethyl caffeate benzoxanthene lignan is a derivative of caffeic acid phenethyl ester that induces bystander autophagy in WiDr cells. Mol. Biol. Rep. 2014, 41, 85-94.

19. Gao, X.M.; Wang, R.R.; Niu, D.Y.; Meng, C.Y.; Yang, L.M.; Zheng, Y.T.; Yang, G.Y.; Hu, Q.F.; Sun, H.D.; Xiao, W.L. Bioactive dibenzocyclooctadiene lignans from the stems of Schisandra neglecta. J. Nat. Prod. 2013, 76, 1052-1057. 
20. Giraldi, T.; Guarino, A.M.; Nisi, C.; Sava, G. Antitumor and antimetastatic effects of benzenoid triazenes in mice bearing lewis lung carcinoma. Pharmacol. Res. Commun. 1980, 12, 1-11.

21. Chang, K.C.; Duh, C.Y.; Chen, I.S.; Tsai, I.L. A cytotoxic butenolide, two new dolabellane diterpenoids, a chroman and a benzoquinol derivative formosan Casearia membranacea. Planta Med. 2003, 69, 667-672.

22. Chen, P.Y.; Wu, J.D.; Tang, K.Y.; Yu, C.C.; Kuo, Y.H.; Zhong, W.B.; Lee, C.K. Isolation and synthesis of a bioactive benzenoid derivative from the fruiting bodies of Antrodia camphorata. Molecules 2013, 18, 7600-7608.

23. Ge, Y.B.; Li, M.S.; Mei, Z.N.; Yang, G.Z. Two new flavonol glycosides from the leaves of Elaeagnus pungens. J. Asian Nat. Prod. Res. 2013. 15, 1073-1079.

24. Lou, F.M.; Yang, J.; Bai, Z.C.; Wu, B.F. Studies on chemical constituents in rhizome of Elaeagnus bockii. Zhongguo Zhong Yao Za Zhi 2006, 31, 988-989.

25. Cao, S.G.; Tanaka, T.; Mizuno, M.; Inoue, K. Flavonol glycosides from Elaeagnus lanceollata (Elaeagnaceae). Nat. Prod. Lett. 2001, 15, 211-216.

26. Song, W.W.; Li, B.; Liu, J.K. A new lignan from Elaeagnus lanceolata (Elaeagnaceae). Acta. Bot. Yunnanica 2010, 32, 455-462.

27. Bendaikha, S.; Gadaut, M.; Harakat, D.; Magid, A. Acylated flavonol glycosides from the flower of Elaeagnus angustifolia L. Phytochemistry 2014, 103, 129-136.

28. Siegel, R.; Naishadham, D.; Jemal, A. Cancer statistics: 2013. CA: A Cancer J. Clin. 2013, 63, 11-30.

29. Bray, F.; Jemal, A.; Grey, N.; Ferlay, J.; Forman, D. Global cancer transitions according to the Human Development Index (2008-2030): A population-based study. Lancet. Oncol. 2012, 13, $790-801$.

30. Tran, Q.N. A novel method for finding non-small cell lung cancer diagnosis biomarkers. BMC Med. Genomics 2013, 6, 11-20.

31. Meoni, G.; Cecere, F.L.; Lucherini, E.; di Costanzo, F. Medical treatment of advanced non-small cell lung cancer in elderly patients: A review of the role of chemotherapy and targeted agents. J. Geriatr. Oncol. 2013, 4, 282-290.

32. Seebacher, W.; Simic, N.; Weis, R.; Saf. R.; Kunert, O. Complete assignments of ${ }^{1} \mathrm{H}$ and ${ }^{13} \mathrm{C}$ NMR resonances of oleanolic acid, $18 \alpha$-oleanolic acid, ursolic acid and their 11-oxo derivatives. Magn. Reson. Chem. 2003, 41, 636-638.

33. Takahashi, H.; Iuchi, M.; Fujita, Y.; Minami, H.; Fukuyama, Y. Coumaroyl triterpenes from Casuarina equisetifolia. Phytochemistry 1999, 51, 543-550.

34. Chen, B.; Duan, H.; Takaishi, Y. Triterpene caffeoyl esters and diterpenes from Celastrus stephanotifolius. Phytochemistry 1999, 51, 683-687.

35. Murphy, B.T.; MacKinnon, S.L.; Yan, X.; Hammond, G.B.; Vaisberg, A.J.; Neto, C.C. Identification of triterpene hydroxycinnamates with in vitro antitumor activity from whole cranberry fruit (Vaccinium macrocarpon). J. Agric. Food Chem. 2003, 51, 3541-3545.

36. Jeong, W.; Hong, S.S.; Kim, N.; Yang, Y.T.; Shin, Y.S.; Lee, C.; Hwang, B.Y.; Lee, D. Bioactive triterpenoids from Callistemon lanceolatus. Arch. Pharm. Res. 2009, 32, 845-849. 
37. Katai, M.; Terai, T.; Meguri, H. Triterpenoids of the bark of Pieris japonica D. DON (Japanese Name: Asebi). II. ${ }^{13} \mathrm{C}$ nuclear magnetic resonance of the $\gamma$-lactones of ursane and oleanane-type triterpenes. Chem. Pharm. Bull. 1983, 31, 1567-1571.

38. Huang, H.; Sun, H.; Zhao, S. Triterpenoids of Isodon loxothyrsus. Phytochemistry 1996, 42, $1665-1666$.

39. Collins, D.O.; Ruddock, P.L.D.; de Grasse, J.C.; Reynolds, W.F.; Reese, P.B. Microbial transformation of cadina-4,10(15)-dien-3-one, aromadendr-1(10)-en-9-one and methyl ursolate by Mucor plumbeus ATCC 4740. Phytochemistry 2002, 59, 479-488.

40. Tijjani, A.; Ndukwe, I.G.; Ayo, R.G. Isolation and characterization of Lup-20(29)-ene-3, 28-diol (Betulin) from the stem-bark of Adenium obesum (Apocynaceae). Trop. J. Pharm. Res. 2012, 11, 259-262.

41. Baek, M.Y.; Cho, J.G.; Lee, D.Y.; Ahn, E.M.; Jeong, T.S.; Baek, N.I. Isolation of triterpenoids from the stem bark of Albizia julibrissin and their inhibition activity on ACAT-1 and ACAT-2. J. Korean Soc. Appl. Biol. Chem. 2010, 53, 310-315.

42. Liu, M.; Yang, S.; Jin, L.; Hu, D.; Wu, Z.; Yang, S. Chemical constituents of the ethyl acetate extract of Belamcanda chinensis (L.) DC roots and their antitumoractivities. Molecules 2012, 17, 6156-6169.

43. Xu, S.; Shang, M.Y.; Liu, G.X.; Xu, F.; Wang, X.; Shou, C.C.; Cai, S.Q. Chemical constituents from the rhizomes of Smilax glabra and their antimicrobial activity. Molecules 2013, 18, 5265-5287.

44. Wei, Y.; Xie, Q.; Dong, W.; Ito, Y. Separation of epigallocatechin and flavonoids from Hypericum perforatum L. by high-speed counter-current chromatography and preparative high-performance liquid chromatography. J. Chromatogr. A 2009, 1216, 4313-4318.

45. Timmers, M.; Urban, S. On-line (HPLC-NMR) and off-line phytochemical profiling of the Australian plant, Lasiopetalum macrophyllum. Nat. Prod. Commun. 2012, 7, 551-560.

46. Liao, C.R.; Ho, Y.L.; Huang, G.J.; Yang, C.S.; Chao, C.Y.; Chang, Y.S.; Kuo, Y.H. One lignanoid compound and four triterpenoid compounds with anti-inflammatory activity from the leaves of Elaeagnus oldhamii maxim. Molecules 2013, 18, 13218-13227.

47. Świsłocka, R.; Kowczyk-Sadowy, M.; Kalinowska, M.; Lewandowski, W. Spectroscopic (FT-IR, FT-Raman, ${ }^{1} \mathrm{H}$ and ${ }^{13} \mathrm{C} \mathrm{NMR}$ ) and theoretical studies of $p$-coumaric acid and alkali metal p-coumarates. Spectroscopy 2012, 27, 35-48.

48. Al-Musayeib, N.; Perveen, S.; Fatima, I.; Nasir, M.; Hussain, A. Antioxidant, anti-glycation and anti-inflammatory activities of phenolic constituents from Cordia sinensis. Molecules 2011, 16, 10214-10216.

49. Jadrijević-Mladar Takac, M.; Vikić Topić, D. FT-IR and NMR spectroscopic studies of salicylic acid derivatives. II. Comparison of 2-hydroxy- and 2,4- and 2,5-dihydroxy derivatives. Acta Pharm. 2004, 54, 177-191.

50. Yoshioka, T.; Inokuchi, T.; Fujioka, S.; Kimura, Y. Phenolic compounds and flavonoids as plant growth regulators from fruit and leaf of Vitex rotundifolia. Z. Naturforsch. C 2004, 59, 509-514.

51. Ngan, L.T.; Moon, J.K.; Shibamoto, T.; Ahn, Y.J. Growth-inhibiting, bactericidal, and urease inhibitory effects of Paeonia lactiflora root constituents and related compounds on antibiotic-susceptible and resistant strains of Helicobacter pylori. J. Agric. Food Chem. 2012, 60, 9062-9073. 
52. Jeon, K.S.; Na, H.J.; Kim, Y.M.; Kwon, H.J. Antiangiogenic activity of 4-O-methylgallic acid from Canavalia gladiata, a dietary legume. Biochem. Biophys. Res. Commun. 2005, 330, 1268-1274.

53. Liu, K.; Guo, L.; Miao, L.; Bao, W.; Yang, J.; Li, X.; Xi, T.; Zhao, W. Ursolic acid inhibits epithelial-mesenchymal transition by suppressing the expression of astrocyte-elevated gene-1 in human nonsmall cell lung cancer A549 cells. Anticancer Drugs 2013, 24, 494-503.

54. Hao, J.; Liu, J.; Wen, X.; Sun, H. Synthesis and cytotoxicity evaluation of oleanolic acid derivatives. Bioorg. Med. Chem. Lett. 2013, 23, 2074-2077.

55. Sun, H.; Fang, W.S.; Wang, W.Z.; Hu, C. Structure-activity relationships of Oleanane- and ursane- type triterpenoids. Bot. Stud. 2006, 47, 339-368.

56. Min, B.S.; Kim, Y.H.; Lee, S.M.; Jung, H.J.; Lee, J.S.; Na, M.K.; Lee, C.O.; Lee, J.P.; Bae, K. Kaempferol-induced growth inhibition and apoptosis in A549 lung cancer cells is mediated by activation of MEK-MAPK. J. Cell Physiol. 2003, 197, 110-121.

57. Maurya, D.K.; Nandakumar, N.; Devasagayam, T.P. Anticancer property of gallic acid in A549, a human lung adenocarcinoma cell line, and possible mechanisms. J. Clin. Biochem. Nutr. 2011, $48,85-90$.

Sample Availability: Samples of the compounds are available from the authors.

(C) 2014 by the authors; licensee MDPI, Basel, Switzerland. This article is an open access article distributed under the terms and conditions of the Creative Commons Attribution license (http://creativecommons.org/licenses/by/3.0/). 